\title{
Synergetic Enhancement of Fluorescence and Magnetic Resonance Signals Assisted by Albumin Cage
}

Lirong Wang, ${ }^{1}$ Qing Wan, ${ }^{1}$ Rongyuan Zhang, ${ }^{6}$ Bo Situ, ${ }^{3}$ Kaiyuan $N i,{ }^{5}$ Jinhao Gao, ${ }^{2}$ Zhiming Wang, ${ }^{1, *}$ Anjun Qin, ${ }^{1, *}$ Ben Zhong Tang ${ }^{1,4}$

Dr. L. Wang, Dr. Q. Wan, Prof. Z. Wang, Prof. A. Qin, Prof. B. Z. Tang

State Key Laboratory of Luminescent Materials and Devices, Guangdong Provincial Key Laboratory of Luminescence from Molecular Aggregates, SCUT-HKUST Joint Research Institute, AIE Institute, Center for Aggregation-Induced Emission, South China University of Technology (SCUT), Guangzhou 510640, China.

E-mail: wangzhiming@scut.edu.cn, msqinaj@scut.edu.cn

Prof. B. Z. Tang

Department of Chemistry, Hong Kong Branch of Chinese National Engineering Research Center for Tissue Restoration and Reconstruction, Institute for Advanced Study, and Department of Chemical and Biological Engineering, The Hong Kong University of Science and Technology, Clear Water Bay, Kowloon, Hong Kong, China.

Prof. J. Gao

Department of Chemical Biology, College of Chemistry and Chemical Engineering, Xiamen University, Xiamen 361005, China.

Dr. B. Situ

Department of Laboratory Medicine, Nanfang Hospital, Southern Medical University, Guangzhou 510515, China.

Dr. K. Ni

Koch Institute for Integrative Cancer Research, Massachusetts Institute of Technology, Cambridge, MA, USA.

Dr. R. Zhang

Department of Urology, The First Affiliated Hospital of Soochow University, 188 Shizi Road, Suzhou 215006, China.

Keywords: albumin cage, fluorescence, magnetic resonance signal, aggregation-induced emission, synergetic enhancement

Bimodal fluorescence and magnetic resonance imaging (FLI/MRI) is important for early diagnosis of malignant tumors. Yet, facile and opportune strategies to synergistically enhance fluorescence intensity and magnetic resonance (MR) contrast effect have been rarely reported. Here, a facile albumin cage (AC) strategy is provided to synergistically enhance the fluorescence intensity by aggregation-induced emission (AIE) and MR contrast with prolonged rotational correlation time $\left(\tau_{\mathrm{R}}\right)$ of $\mathrm{Gd}(\mathrm{III})$ chelates and diffusion correlation time $\left(\tau_{\mathrm{D}}\right)$ of surrounding water molecules. The amphiphilic bimodal FLI/MRI probe of NGd, could be facilely loaded into ACs to generate supramolecular structure of NGd-albumin cages (NGdACs), which show excellent biocompatibility and biosafety, and exhibit superior fluorescence 
quantum yield and $r_{1}$ over NGd with 6- and 8-fold enhancement, respectively. Moreover, compared with clinical MRI contrast agent of Gd-DOTA, $r_{1}$ of NGd-AC shows 17-fold enhancement. As a result, NGd-ACs successfully elicit high-performance bimodal FLI/MRI in vitro and brighter MR signals are observed in liver and tumor after intravenous injection of NGd-ACs with a dosage of $6 \mu \mathrm{mol} \mathrm{Gd}(\mathrm{III}) / \mathrm{kg}$ body weight. This strategy is generic and feasible, and successfully realizes a " $1+1>2$ ” effect for dual-modal FLI/MRI.

\section{Introduction}

Cancer incidence and mortality are rapidly growing and expected to rank as the leading cause of death in the world. ${ }^{[1]}$ In order to fight against cancer, it is particularly urgent and important to develop accurate diagnostic techniques to detect lesions as early as possible. Multimodal imaging plays an important role in early and accurate diagnosis of malignant tumors. ${ }^{[2]}$ Among the widely used imaging techniques, fluorescence imaging (FLI) shows high sensitivity but is limited with tissue-penetration of light for deep-seated tumors. In stark control, magnetic resonance imaging (MRI) stands out by virtue of non-ionization, unlimited tissue-penetration depth, and high spatial resolution, while is hurdled by low sensitivity due to abundant background signal. Integrating FLI and MRI to realize bimodal FLI/MRI can benefit each other, which arouses great interests among researchers to develop novel bimodal FLI/MRI probes. ${ }^{[3]}$

FLI/MRI bimodal probes can be simply constructed by conjugating/coupling various fluorescent moieties with Gd chelates. ${ }^{[4]}$ For MR contrast, Gd chelates mainly contribute to $T_{1}$ contrast effect and their contrast ability can be quantified by the relaxivity $\left(r_{1}\right),{ }^{[5]}$ which is positively associated to rotational correlation time $\left(\tau_{\mathrm{R}}\right)$ of contrast agents and diffusion correlation time $\left(\tau_{\mathrm{D}}\right)$ of water molecules around contract agents. ${ }^{[6]}$ Therefore, it is very important to guarantee that Gd chelates contained in the FLI/MRI bimodal probes are able to easily get access to water molecules in the physiological conditions to enhance the contrast 
effect, but the fluorescence intensity of FLI/MRI bimodal probe is usually hammered with notorious aggregation-caused quenching (ACQ) effect in physiological conditions. Aggregation-induced emission (AIE) with the mechanism of restriction of intramolecular motion (RIM) is a meaningful strategy to address this problem. ${ }^{[7]}$ Combining Gd chelates with AIE moieties (AIE-Gd) into amphiphilic bimodal probes would not only enhance the fluorescence intensity but also improve the relaxivity with prolonged $\tau_{\mathrm{R} .}{ }^{[4 \mathrm{a}]}$ Unfortunately, due to the strong hydrophobicity of AIE moieties, the Gd chelates are easily embedded inside the tightly packed aggregates, and couldn't easily get access to ambient water. Opposite strategies for constructing Gd chelates on the outside and AIE moiety on the inside need to be precise designed, which is strongly limited by the properties of substrates and preparation process, making them be not universal and practical. ${ }^{[4 a, 8]}$ Therefore, to satisfy the constraint of intramolecular motion in hydrophilic environment, we proposed that an amphiphilic structure with geometrically confined space would be ideal to load AIE-Gd bimodal probes for this purpose. Thanks to the hydrophobic interaction and steric hindrance, the intramolecular motion of AIE moieties could be restricted to enhance the fluorescence for FLI. Meanwhile, compared with single molecule, the larger size of nanoaggregates with longer $\tau_{\mathrm{R}}$ would strongly improve the relaxivity for MRI. Additionally, the geometrically confined structure of these nanoaggregates facilitate water molecules in and out with slow diffusion rate, that is, prolonged $\tau_{\mathrm{D}}$ of water molecules, which would further contribute to the improvement of relaxivity (Scheme 1).

For the amphiphilic structures, albumin would be the best choice with incomparable advantages, such as biocompatibility and biodegradability. ${ }^{[9]}$ Different albumin-based biomaterials with desired properties have been developed over the past few decades. ${ }^{[10]}$ By virtue of amphiphilic structure, albumin has been widely reported as superb nanocarriers to either improve the relaxivity ${ }^{[6 a, 11]}$ or enhance the fluorescence intensity based on RIM of AIE moieties. ${ }^{[12]}$ Inspiringly, we design an amphiphilic FLI/MRI dual-modal probe of NGd by 
combination of AIE moiety and Gd-DOTA, which could closely bind to bovine serum albumin (BSA). And then, we constructed NGd-Albumin Cages (NGd-ACs) through desolvation and glutaraldehyde coupling BSA to load NGd. Compared with NGd, the fluorescence quantum yield and $r_{1}$ of NGd-ACs had a more than 6- and 8-fold improvement, respectively, and their $r_{1}$ was almost 17 -fold higher than that of clinical contrast agent GdDOTA. The phantom images had proven that NGd-ACs showed brighter signals than NGd at the same concentration. Moreover, the results of cell phantom imaging and confocal laser scanning microscope (CLSM) verified that NGd-ACs could be internalized more by HeLa cells and had successfully achieved dual-modal FLI/MRI in vitro. Furthermore, in vivo $T_{1^{-}}$ weighted MR imaging indicated that NGd-ACs had superior ability to contrast the liver and tumor with 5-fold lower dosage than previously reported MR contrast agents. Additionally, biodistribution study showed that NGd-ACs were mainly excreted by hepatobiliary metabolism, and the pathological test certificated that they had good biosafety in vivo. We propose that the "Albumin Cage" structure would be an efficient strategy to achieve the synergetic enhancement of fluorescence intensity and MR contrast effect by combination of AIE mechanism and prolonging the $\tau_{\mathrm{R}}$ of Gd chelates and $\tau_{\mathrm{D}}$ of water molecules around $\mathrm{Gd}$ chelates.

\section{Result and discussion}

\subsection{Synthesis and Characterization of NGd-ACs}

The donor-acceptor (D-A) approach was employed to build the fluorescent moiety, in which dimethoxytriphenylamine and $2 \mathrm{H}$-naphtho[2,3-d] triazole core (NT) were used as the electron-donating and accepting units, respectively. With the introduction of undecylic acid to the triazole part, Gd-DOTA was conjugated into luminogens to obtain the FLI/MRI bimodal probe of NGd. The synthesis and characterizations of NGd in detail were shown in the Supporting Information (Scheme S1 and Figure 1a and S1-S7). NGd shows an absorption maximum at $435 \mathrm{~nm}$ and fluorescent peak at $570 \mathrm{~nm}$ in tetrahydrofuran (THF) (Figure 1b). 
Then, we studied its AIE effect by varying the water fraction $\left(f_{\mathrm{w}}\right)$ in dimethyl sulfoxide (DMSO)/water mixtures (Figure 1c and 1d). The emission intensity decreases with addition of water into DMSO until $f_{\mathrm{w}}$ reaches $20 \%$, because of the twist intramolecular charge transfer (TICT) process. With the increasing of $f_{\mathrm{w}}$ from 20 to $70 \%$, the fluorescence intensity is gradually enhanced, showing a typical AIE feature, but when $f_{\mathrm{w}}$ is higher than $70 \%$, the fluorescence intensity decreases. This might be ascribed to the formation of aggregates with large sizes, which sink to the bottom and decrease the effective concentration of NGd in the detection beam zone. ${ }^{[13]}$ This was also proved by the decrease in relaxation rate of NGd in the DMSO/water mixtures when $f_{\mathrm{w}}$ is higher than $50 \%$ (Figure S8). Notably, introduction of GdDOTA into AIE moiety didn't affect its AIE property (Figure S9). Due to the amphiphilic characteristics of NGd, the critical micelle concentration (CMC) of NGd was detected to be $25 \mu \mathrm{M}$ (Figure S10) by using emission intensity. This reveals that NGd molecules are prone to form aggregates.

To ensure the interaction between NGd molecules and BSA, their binding affinity was firstly estimated by the proton relaxation enhancement. ${ }^{[14]}$ The result demonstrated a high affinity between NGd and BSA with a binding constant of $\sim 1.2 \times 10^{5} \mathrm{M}^{-1}$, and the relaxation rate reaches maximum at the molar ratio of $\mathrm{NGd} / \mathrm{BSA}=2: 1$ (Figure $\mathrm{S} 11$ ). When NGd were added into BSA aqueous solution, the NGd-BSA complexes would form aggregates. In order to stabilize the disbanded structure of aggregates with suitable size, desolvation and glutaraldehyde coupling were applied to synthesize the NGd-Albumin Cages (NGd-ACs) with the initial feed malar ratio of $\mathrm{NGd} / \mathrm{BSA}=2: 1$ (Figure 1e). And the encapsulation efficiency of NGd is deduced to be $c a .35 \%$. Due to the low CMC, parts of NGd molecules are prone to form aggregates, partially hindering NGd molecules to interact with BSA. The dynamic light scattering (DLS) shows the hydrodynamic diameter of NGd-ACs is about $178 \mathrm{~nm}$, which is similar with the result of $\sim 167 \mathrm{~nm}$ measured by transmission electron microscopy (TEM) (Figure 1f). In addition, NGd-ACs are stable in deionized (DI) water, phosphate buffer 
solution (PBS), and fetal bovine serum (FBS) (Figure 1g), indicating their high stability in physiological environment, which is feasible for biological applications.

\subsection{Synergetic Enhancement of fluorescence and MR signals by NGd-ACs in solution state}

To check whether these albumin cages could enhance the fluorescence intensity, the optical properties of NGd-ACs were explored. As shown in Figure 2a and S15a, the fluorescence intensity of NGd-ACs is 7- and 5-time higher than that of NGd in DMSO or water, and the fluorescence quantum yield $\left(\Phi_{\mathrm{F}}\right)$ of NGd-ACs is almost 6 times higher than that of NGd in water or BSA aqueous solution, and more than 3 times higher than that of NGd in DMSO (Figure 2a and Table S1). The obviously enhanced fluorescence intensity and $\Phi_{\mathrm{F}}$ of NGd-ACs should be attributed to the RIM of NGd by inserting into BSA cages (Figure 2c). Meanwhile, the fluorescence intensities of NGd in DMSO/water mixture with $f_{\mathrm{w}}$ of $97 \%$ and DMSO were almost at the same level, but much lower than that of NGd in DMSO/water mixture with $f_{\mathrm{w}}$ of $70 \%$ (Figure $\mathrm{S} 15 \mathrm{~b}$ ). The reason might be that when $f_{\mathrm{w}}$ in the mixture solvent is larger than $70 \%$, the aggregate sediments of NGd become larger and more, further decreasing the effective detection concentration, which leads to the plateau for fluorescence intensity improvement. Because the aggregation of NGd hinders them to interact with BSA effectively, the fluorescence intensity wasn't enhanced even after addition BSA (Figures 2a, 2c, and S12). In addition, the emission peak has an obvious blue-shift for NGd-ACs compared with that of NGd in DMSO (Figure S12a), which is attributed to the noncovalent binding between NGd and BSA through hydrophobic interactions and ion pairing between the cationic groups of gadolinium complex and amino acid residues. ${ }^{[12 c]}$ Surprisingly, the fluorescence intensity of NGd-ACs is slightly weaker than that of NGd in DMSO/water mixture with $f_{\mathrm{w}}$ of $70 \%$ (Figure S12b). This result indicates that the packing of NGd-ACs was not as tight as that of NGd aggregates in water. The slackened structure of NGd-ACs could generate geometrically 
confined effect for permitting the water molecules in and out slowly, hence prolonging $\tau_{\mathrm{D}}$ of the water molecules, which is beneficial to the improvement of MR contrast effect.

Hereafter, MR contrast effect of NGd-ACs was evaluated and compared with NGd molecules in water or BSA aqueous solution, and clinical contrast agent Gd-DOTA (Figure $2 \mathrm{~b}$ and S13a). The $r_{1}$ of NGd-ACs is $85.1 \pm 7.0 \mathrm{mM}^{-1} \mathrm{~s}^{-1}$ at $0.5 \mathrm{~T}$, which is 17 -fold and nearly 8-fold higher than that of Gd-DOTA $\left(4.9 \pm 0.5 \mathrm{mM}^{-1} \mathrm{~s}^{-1}\right)$ and NGd in aqueous solution $(10.9 \pm$ $\left.1.2 \mathrm{mM}^{-1} \mathrm{~s}^{-1}\right)$, respectively. This result verifies that the strategy of albumin cage could largely improve the relaxivity of contrast agents. Such a high relaxivity should be ascribed to the elongated $\tau_{\mathrm{R} 2}$ of large size of nanoparticles and $\tau_{\mathrm{D} 2}$ of water molecules trapped in the interval of BSA cages $\left(\tau_{\mathrm{R} 1}<\tau_{\mathrm{R} 2}\right.$ and $\tau_{\mathrm{D} 1}<\tau_{\mathrm{D} 2}$, Figure $\left.2 \mathrm{c}\right)$. In addition, the relaxivity of NGd in aqueous solution is higher than that of Gd-DOTA, due to the high molecular weight of NGd and its aggregate state in water. ${ }^{[4 a]}$ We further dispersed NGd into the BSA aqueous solution with the molar ratio of 2:1, of which the relaxivity is up to $28.4 \pm 1.0 \mathrm{mM}^{-1} \mathrm{~s}^{-1}, 2$ times higher than that of NGd in water, but is still 3-fold lower than that of NGd-ACs (Figures 2b and S13a). This consequence could be attributed to the weak interaction of NGd aggregates (slightly elongated $\left.\tau_{\mathrm{R} 3}\right)$ with BSA and the slightly prolonged $\tau_{\mathrm{D} 3}$ of water molecules because of the "waterblocking" function of albumin $\left(\tau_{\mathrm{R} 1}<\tau_{\mathrm{R} 3}<\tau_{\mathrm{R} 2}\right.$ and $\tau_{\mathrm{D} 1}<\tau_{\mathrm{D} 3}<\tau_{\mathrm{D} 2}$, Figure $\left.2 \mathrm{c}\right)$. Furthermore, the phantom images of these four groups also prove that NGd-ACs have the brightest contrast effect compared with three other circumstances at the same $\mathrm{Gd}(\mathrm{III})$ concentrations (Figure $2 \mathrm{~d}$ and S13b). From the phantom images, it can be seen that NGd-ACs at a low Gd(III) concentration $(0.025 \mathrm{mM})$ can reach the same brightness of Gd-DOTA at a 16-fold higher concentration $(0.4 \mathrm{mM})$ (Figure S13b), which indicates that a lower dose can generate satisfied effect for NGd-ACs. Therefore, the results of relaxivity and $T_{1}$-weighted images illustrate that these albumin cages loading NGd can also largely improve MR contrast effect. Combining with the enhanced fluorescence intensity, NGd-ACs would have great potential as a high-performance FLI/MRI bimodal probe for in vitro imaging. 


\subsection{In vitro bimodal FLI/MRI of NGd-ACs}

Subsequently, bimodal FLI/MRI of NGd-ACs in vitro was explored. $T_{1}$-weighted MR images of HeLa cells incubated with NGd-ACs, NGd, and Gd-DOTA with the same Gd(III) concentration of $100 \mu \mathrm{M}$ were acquired. As shown in Figure 3a and 3b, the cells treated with NGd-ACs exhibit strongest positive contrast signal among with those of NGd, Gd-DOTA and PBS groups. Meanwhile, fluorescence intensity of cells incubated with NGd-ACs is brighter than that with NGd. Because Gd-DOTA and PBS are non-emissive, no fluorescence signal is found in the cells treated with them. These results indicate that NGd-ACs can realize in vitro bimodal FLI/MRI. To quantify the amount of these probes per cell, inductively coupled plasma mass spectroscopy (ICP-MS) was employed (Figure 3c). The cellular uptake of NGdACs, NGd and Gd-DOTA was about $195.6 \pm 5.3,116.1 \pm 4.8,74.5 \pm 8.5 \mathrm{fg} \mathrm{Gd(III)/cell,}$ respectively. Furthermore, CLSM was employed to observe intracellular accumulation of NGd-ACs and NGd. Obviously, NGd-ACs have been taken up more than NGd by HeLa cells (Figure 3d), illustrating NGd-ACs have a higher cell uptake than other molecules. These results could be attributed to the gp60 albumin receptor distributing on cell surfaces, which can bind albumins to induce clustering of gp60-albumin and association with caveolin-1 and further complete the transcytosis process. ${ }^{[9 b]}$ Moreover, 3-(4,5-dimerthylthiazol-2-yl)-2,5diphenyltetrazolium bromide (MTT) assay showed that HeLa cells had a 100\% viability after incubated with NGd-ACs for 24 h even at a Gd(III) concentration of $100 \mu \mathrm{M}$, while only $70 \%$ viability was obtained when incubated with NGd (Figure S14). This result suggests that NGdACs have better biocompatibility than NGd.

\subsection{In vivo MRI of NGd-ACs}

With super performance in $T_{1}$-weighted contrast ability in solution state, NGd-ACs were intravenously injected with a low dose of $6 \mu \mathrm{mol} \mathrm{Gd(III)/kg} \mathrm{body} \mathrm{weight} \mathrm{for} \mathrm{in} \mathrm{vivo} \mathrm{imaging.}$ $T_{1}$-weighted contrast capability of NGd-ACs in liver and tumor of tumor-bearing mice was explored. Clinical contrast agent Gd-DOTA was used as a comparison. $T_{1}$-weighted images at 
different time points of pre-injection, $1,3,7$, and $12 \mathrm{~h}$ were collected on a $1 \mathrm{~T}$ MRI scanner. As shown in Figure 4a, compared with per-injection, it can be easily seen a bright signal in liver over time with a peak at $3 \mathrm{~h}$ post-injection, and the signal weakened at $12 \mathrm{~h}$ postinjection. While for the control group with the same Gd(III) content, slightly bright signal was observed at $1 \mathrm{~h}$ post-injection and disappeared quickly due to the rapid renal clearance and weak contrast effect of Gd-DOTA. Subsequently, we analyzed the contrast capability of NGdACs in the tumor region. As illustrated in Figure 4b, the positive signals of tumor are getting brighter than that of pre-injection and peak after injection for $7 \mathrm{~h}$. In contrast, there are little positive signals can be seen at different time point after injection of Gd-DOTA apart from inferior bright signal after injection for $1 \mathrm{~h}$. In addition, we also used the values of $\mathrm{SNR}_{\text {post }} / \mathrm{SNR}_{\text {pre }}{ }^{[15]}$ and $\mathrm{CNR}^{[4 \mathrm{c}, 16]}$ to quantitatively evaluate the change of brightness in liver and tumor. In the liver, the $\mathrm{SNR}_{\text {post }} / \mathrm{SNR}_{\text {pre }}$ values of $T_{1}$-weighted images are deduced to be $1.6 \pm 0.2,2.7 \pm 0.1,2.1 \pm 0.1,1.4 \pm 0.1$ at $1,3,7$, and $12 \mathrm{~h}$ after administration of NGd-ACs, respectively (Figure $4 \mathrm{c}$ ). Moreover, the peak signal after injection for $3 \mathrm{~h}$ is $\sim 2.7$-fold higher

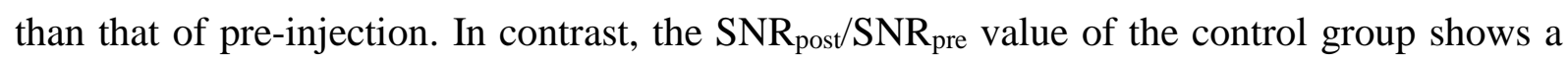
peak of $1.2 \pm 0.1$ after injection for $1 \mathrm{~h}$, which is more than 2 -fold lower than that after injection of NGd-ACs group for $3 \mathrm{~h}$. While the highest CNR for tumor of NGd-ACs after injection for $7 \mathrm{~h}$ is $\sim 56 \%$, which is almost 6 -fold and more than 3 -fold higher than that of prescan $(\sim 9 \%)$ and the control group after injection for $1 \mathrm{~h}(\sim 17 \%)$, respectively (Figure $4 \mathrm{~d})$. These results suggest that NGd-ACs hold potential for $T_{1}$-weighted tumor imaging because of their large size, which could make them accumulated and retained in the tumor by enhanced permeability and retention (EPR) effect. ${ }^{[17]}$ Importantly, the decreased signals of liver and tumor indicate that NGd-ACs could be gradually cleared from the body after injection for 12 h.

\subsection{Biodistribution and biosafety of NGd-ACs}


The biodistribution of NGd-ACs in tumor-bearing mice were explored at different time intervals with a dose of $30 \mu \mathrm{mol} \mathrm{Gd(III)/kg} \mathrm{body} \mathrm{weight.} \mathrm{As} \mathrm{shown} \mathrm{in} \mathrm{Figure} \mathrm{5a,} \mathrm{the} \mathrm{liver}$ exhibits the strongest fluorescence signal among these tissues, and intestine, lung, and tumor show faint fluorescence after injection for $3 \mathrm{~h}$. However, after injection for $12 \mathrm{~h}$, the strongest signals were observed in intestine and liver, and no signal was found on the other tissues and tumor. To acquire a quantitative assessment of biodistribution of NGd-ACs, heart, liver, spleen, lung, kidney, intestine and tumors were collected and analyzed for Gd(III) content by ICP-MS. Unlike the ex vivo fluorescence signals, NGd-ACs have the highest accumulation in spleen, liver, intestine followed by tumor, lung and kidney (Figure 5b). High accumulation of NGd-ACs in the spleen and liver could be attributed to reticuloendothelial system (RES), ${ }^{[18]}$ whereas enrichment in the intestine likely signifies the clearance of nanoparticles. Surprisingly, with similarly high contents of NGd-ACs in spleen and liver after injection for 3 $\mathrm{h}$ (Figure 5b), no fluorescent signal was observed in spleen but strong fluorescent signal could be detected in liver (Figure 5a). This could be ascribed to the different disparities in light absorption and scattering between liver and spleen. ${ }^{[4 c, 19]}$ Uptake by tumor indicates again that the large size of NGd-ACs extends their residence time in the body, which is conducive to the EPR effect. All organs show significantly decreased Gd(III) content after $12 \mathrm{~h}$, further suggesting the clearance of NGd-ACs. These data confirm that NGd-ACs are mainly taken up by RES and eliminated over time probably by hepatobiliary metabolism. ${ }^{[20]}$

To evaluate long-term biosafety of NGd-ACs, the serological and histological examinations were carried out. NGd-ACs were injected into healthy BALB/c mice via tail vein at a higher dosage of $30 \mu \mathrm{mol} \mathrm{Gd(III)/kg} \mathrm{body} \mathrm{weight.} \mathrm{For} \mathrm{comparison,} \mathrm{PBS} \mathrm{buffer} \mathrm{with} \mathrm{the} \mathrm{same}$ volume was used under the same experimental conditions. After one month, these mice were all executed and dissected to collect the blood and main organs. The alanine aminotransferase (ALT) and aspartate aminotransferase (AST) are typical serum biochemical indexes. As shown in Figure 5c, these two indexes of mice treated by NGd-ACs are on the similar level to 
those of PBS group, suggesting that NGd-ACs have no effect on liver function. Moreover, hematoxylin and eosin (H\&E) stained images show that there is no obvious inflammation or cell necrosis in main organs (Figure 5d), indicating that NGd-ACs have good biosafety and will facilitate their practical applications.

\section{Conclusion}

A facile and straightforward strategy to synergistically enhance $\Phi_{\mathrm{F}}$ and $r_{1}$ by albumin caged bimodal FLI/MRI probe of NGd-ACs was presented. This probe had good biocompatibility and AIE activity by combination of AIE mechanism (i.e. RIM) and prolonging the $\tau_{\mathrm{R}}$ of $\mathrm{Gd}(\mathrm{III})$ chelates and $\tau_{\mathrm{D}}$ of surrounding water molecules. Compared with NGd and clinical contrast agent Gd-DOTA, NGd-ACs exhibited remarkable improvement in both fluorescence and $T_{1}$-weighted contrast, which endowed a high-performance bimodal FLI/MRI in vitro. As a result, in vivo MRI can be acquired using NGd-ACs with a lower dosage than most reported probes. This albumin-cage strategy realizes a " $1+1>2$ " effect for bimodal FLI/MRI, which hopefully inspires researchers to develop biocompatible nearinfrared (NIR) or NIR-II FLI/MRI dual-modal probes for the accurate diagnosis and surgical navigation.

\section{Supporting Information}

Supporting Information is available from the Wiley Online Library or from the author.

\section{Acknowledgements}

L.W. and Q.W. contributed equally to this work. The authors are grateful for financial support from the National Natural Science Foundation of China (21788102, 21525417, and 51620105009), the Natural Science Foundation of Guangdong Province (2019B030301003, 2019A1515012144 and 2016A030312002), the National Key Research and Development Program of China (Intergovernmental cooperation project, 2017YFE0132200) and the Innovation and Technology Commission of Hong Kong (ITC-CNERC14S01).

Received: ((will be filled in by the editorial staff))

Revised: ((will be filled in by the editorial staff)) Published online: ((will be filled in by the editorial staff)) 
References

[1] F. Bray, J. Ferlay, I. Soerjomataram, R. L. Siegel, L. A. Torre, A. Jemal, CA Cancer J. Clin. 2018, 68, 394.

[2] a) M. L. James, S. S. Gambhir, Physiol. Rev. 2012, 92, 897; b) D. S. Karaman, M. P. Sarparanta, J. M. Rosenholm, A. J. Airaksinen, Adv. Mater. 2018, 30, e1703651.

[3] a) V. S. Talanov, C. A. Regino, H. Kobayashi, M. Bernardo, P. L. Choyke, M. W. Brechbiel, Nano Lett 2006, 6, 1459; b) R. Yan, Y. Hu, F. Liu, S. Wei, D. Fang, A. J. Shuhendler, H. Liu, H. Y. Chen, D.Ye, J. Am. Chem. Soc. 2019, 141, 10331.

[4] a) H. Li, G. Parigi, C. Luchinat, T. J. Meade, J. Am. Chem. Soc. 2019, 141, 6224; b) C.

Rivas, G. J. Stasiuk, J. Gallo, F. Minuzzi, G. A. Rutter, N. J. Long, Inorg. Chem. 2013, 52, 14284. c) V. S. Harrison, C. E. Carney, K. W. MacRenaris, E. A. Waters, T. J. Meade, J. Am. Chem. Soc. 2015, 137, 9108.

[5] J. Wahsner, E. M. Gale, A. Rodriguez-Rodriguez, P. Caravan, Chem. Rev. 2019, 119, 957.

[6] a) L. Wang, H. Lin, L. Ma, J. Jin, T. Shen, R. Wei, X. Wang, H. Ai, Z. Chen, J. Gao, Nanoscale 2017, 9, 4516; b) K. Ni, Z. Zhao, Z. Zhang, Z. Zhou, L. Yang, L. Wang, H. Ai, J. Gao, Nanoscale 2016, 8, 3768; c) A. Gizzatov, C. Stigliano, J. S. Ananta, R. Sethi, R. Xu, A. Guven, M. Ramirez, H. Shen, A. Sood, M. Ferrari, Cancer Lett. 2014, 352, 97; d) J. S. Ananta, B. Godin, R. Sethi, L. Moriggi, X. Liu, R. E. Serda, R. Krishnamurthy, R. Muthupillai, R. D. Bolskar, L. Helm, M. Ferrari, L. J. Wilson, P. Decuzzi, Nat.Nanotechnol. 2010, 5, 815; e) J. Hu, S. Liu, Sci. China Chem. 2018, 61, 1110.

[7] a) D. Wang, B. Z. Tang, Acc. Chem. Res. 2019, 52, 2559; b) H.-T. Feng, J. W. Y. Lam, B. Z. Tang, Coord. Chem. Rev. 2020, 406, 213142; c) J. Li, J. Wang, H. Li, N. Song, D. Wang, B. Z. Tang, Chem. Soc. Rev. 2020, 49, 1144; d) X. Cai, B. Liu, Angew.Chem. Int. Ed. 2020, 59, 9868; e) H. Zhang, J. Liu, L. Du, C. Ma, N. L. C. Leung, Y. Niu, A. Qin, J. Sun, Q. Peng, H. H. Y. Sung, I. D. Williams, R. T. K. Kwok, J. W. Y. Lam, K. S. Wong, D. L. Phillipsd, B. Z. Tang, Mater. Chem. Front. 2019, 3, 1143. 
[8] Y. Chen, M. Li, Y. Hong, J. W. Y. Lam, Q. Zheng, B. Z. Tang, ACS Appl. Mater. Intefaces 2014, 6, 10783.

[9] a) F. Kratz, J. Control. Release 2008, 132, 171; b) D. Sleep, J. Cameron, L. R. Evans, Biochim. Biophys. Acta. 2013, 1830, 5526.

[10] a) X. Liu, C. Wang, Z. Liu, Adv. Healthc. Mater. 2018, 7, e1800913; b) Z. Liu, X. Chen, Chem. Soc. Rev. 2016, 45, 1432.

[11] a) P. Caravan, Acc. Chem. Res. 2009, 42, 851; b) E. Boros, P. Caravan, J. Med. Chem. 2013, 56, 1782.

[12] a) W. Qin, D. Ding, J. Liu, W. Z. Yuan, Y. Hu, B. Liu, B. Z. Tang, Adv. Funct. Mater. 2012, 22, 771; b) S. Gao, G. Wei, S. Zhang, B. Zheng, J. Xu, G. Chen, M. Li, S. Song, W. Fu, Z. Xiao, W. Lu, Nat. Commun. 2019, 10, 2206; c) S. Wang, F. Hu, Y. Pan, L. G. Ng, B. Liu, Adv. Funct. Mater. 2019, 29, 1902717.

[13] T. He, N. Niu, Z. Chen, S. Li, S. Liu, J. Li, Adv. Funct. Mater. 2018, 28, 1706196.

[14] A. C. Esqueda, J. A. Lopez, G. Andreu-de-Riquer, J. C. Alvarado-Monzon, J. Ratnakar, A. J. Lubag, A. D. Sherry, L. M. De Leon-Rodriguez, J. Am. Chem. Soc. 2009, 131, 11387.

[15] a) L. Wang, H. Lin, X. Chi, C. Sun, J. Huang, X. Tang, H. Chen, X. Luo, Z. Yin, J. Gao, Small 2018, 14, e1801612; b) Z. Zhou, L. Wang, X. Chi, J. Bao, L. Yang, W. Zhao, Z. Chen, X. Wang, X. Chen, J. Gao, ACS Nano 2013, 7, 3287.

[16] Z. Zhou, D. Huang, J. Bao, Q. Chen, G. Liu, Z. Chen, X. Chen, J. Gao, Adv. Mater. 2012, $24,6223$.

[17] a) H. Kang, S. Rho, W. R. Stiles, S. Hu, Y. Baek, D. W. Hwang, S. Kashiwagi, M. S. Kim, H. S. Choi, Adv. Healthc. Mater. 2020, 9, e1901223; b) K. Greish, Methods Mol. Biol. 2010, 624, 25 .

[18] K. Yang, J. Wan, S. Zhang, Y. Zhang, S. T. Lee, Z. Liu, ACS Nano 2011, 5, 516.

[19] N. Sharma, M. A. Saifi, S. B. Singh, C. Godugu, Nanotoxicity 2020, 41.

[20] M. Longmire, P. L. Choyke, H. Kobayashi, Nanomedicine 2008, 3, 703. 


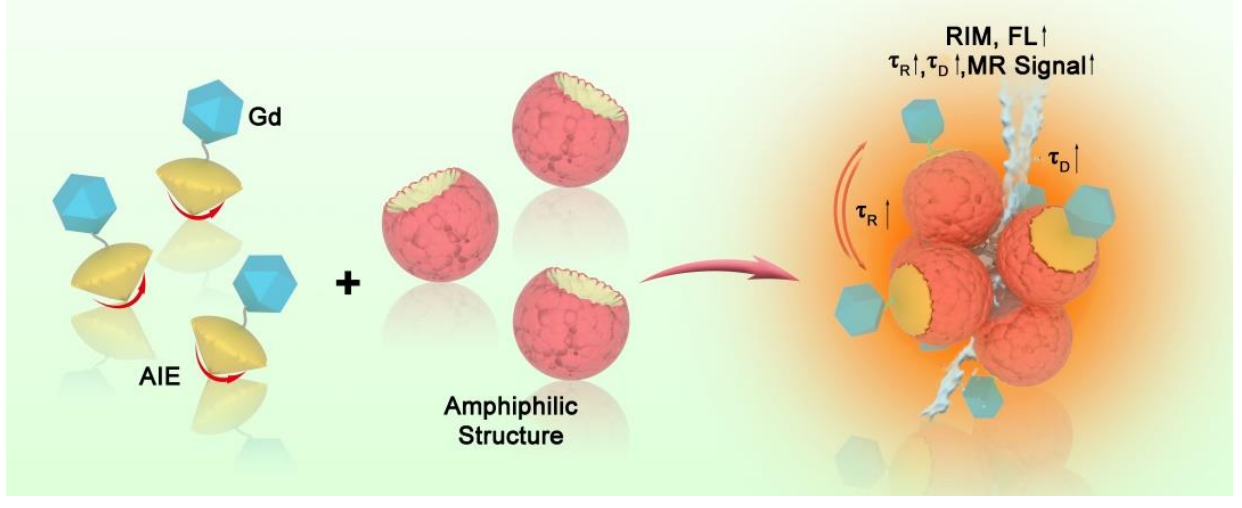

Scheme 1. Schematic illustration of the amphiphilic structures loaded with AIE-Gd molecules that generate supramolecular nanoconstructs with enhanced fluorescence emission and improved MR signal. 

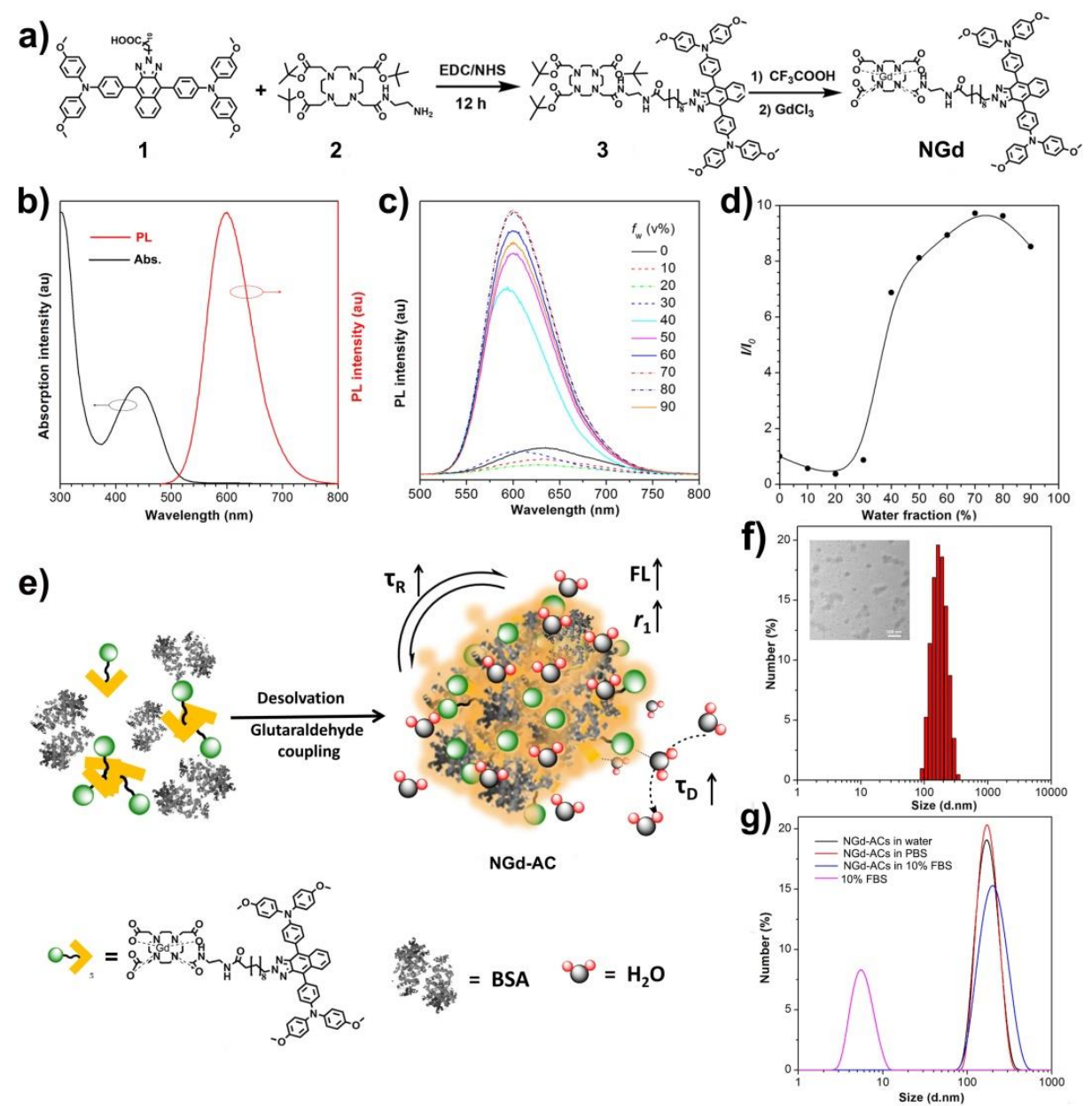

Figure 1. (a) Synthetic route to NGd: 1) deprotection of the tert-butyl groups with trifluoroacetic acid $\left(\mathrm{CF}_{3} \mathrm{COOH}\right)$, and then 2) coordination with $\mathrm{GdCl}_{3}$. (b) Absorption and photoluminescence (PL) spectra of NGd in THF. (c) PL spectra of NGd $(10 \mu \mathrm{M})$ in DMSO/water mixtures with different water fraction $\left(f_{\mathrm{w}}\right)$. (d) Plots of the relative emission intensity of NGd versus water fraction. $I_{0}$ and $I$ are the peak values of PL intensities of NGd $(10 \mu \mathrm{M})$ in DMSO/water mixtures with different $f_{\mathrm{w}}$. (e) Schematic illustration of the synthesis of NGd-Albumin Cage (NGd-AC) and the mechanism of synergistic FLI/MRI enhancement. (f) Hydrodynamic diameter of NGd-ACs in water measured by DLS. Inset: TEM images of NGd-ACs. (g) DLS results of NGd-ACs in DI water, PBS, and 10\% FBS, respectively, and $10 \%$ FBS alone. 

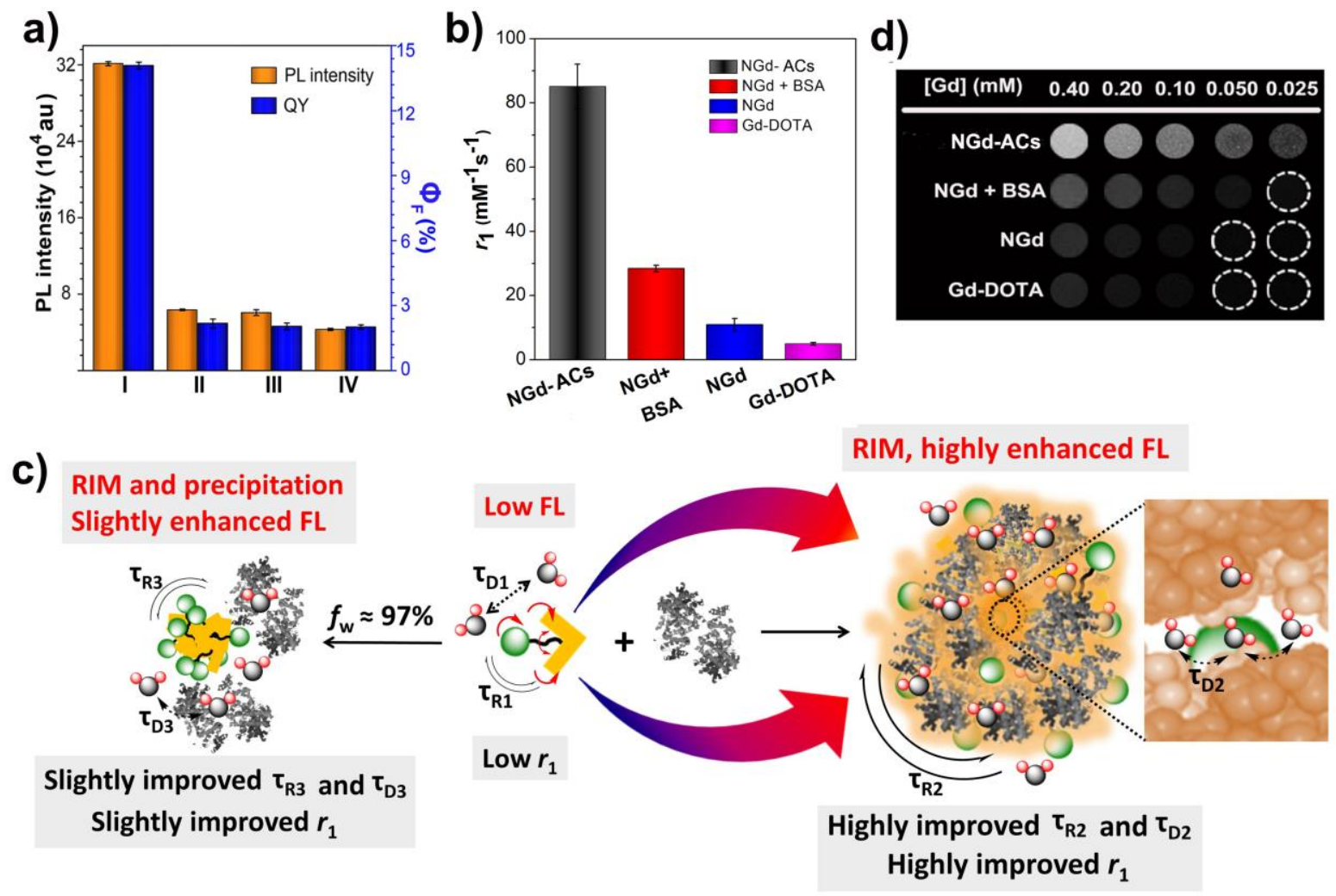

Figure 2. Exploration of fluorescence and relaxation effect. (a) PL intensity and fluorescence quantum yield $\left(\Phi_{\mathrm{F}}\right)$ of (I) NGd-ACs and (II) NGd molecules in BSA solution (the molar ratio of NGd/BSA $=2: 1$ ), (III) water, and (IV) DMSO, respectively. (b) $T_{1}$ relaxivities of NGd-ACs, NGd in BSA aqueous solution (the molar ratio of NGd/BSA = 2:1), NGd in water, and GdDOTA at $0.5 \mathrm{~T}$, respectively. (c) Illustration of mechanism of simultaneous enhancement of fluorescence and relaxivity. $\tau_{\mathrm{R} 1}, \tau_{\mathrm{R} 2}$, and $\tau_{\mathrm{R} 3}$ refer to rotational correlation time of NGd molecules, NGd-ACs, and NGd aggregates in BSA aqueous solution, respectively; $\tau_{\mathrm{D} 1}, \tau_{\mathrm{D} 2}$, and $\tau_{\mathrm{D} 3}$ refer to diffusion correlation time of water molecules around them, respectively. (d) Phantom images of NGd-ACs, NGd in BSA aqueous solution, NGd in water, and Gd-DOTA at different $\mathrm{Gd}(\mathrm{III})$ concentrations, respectively. 

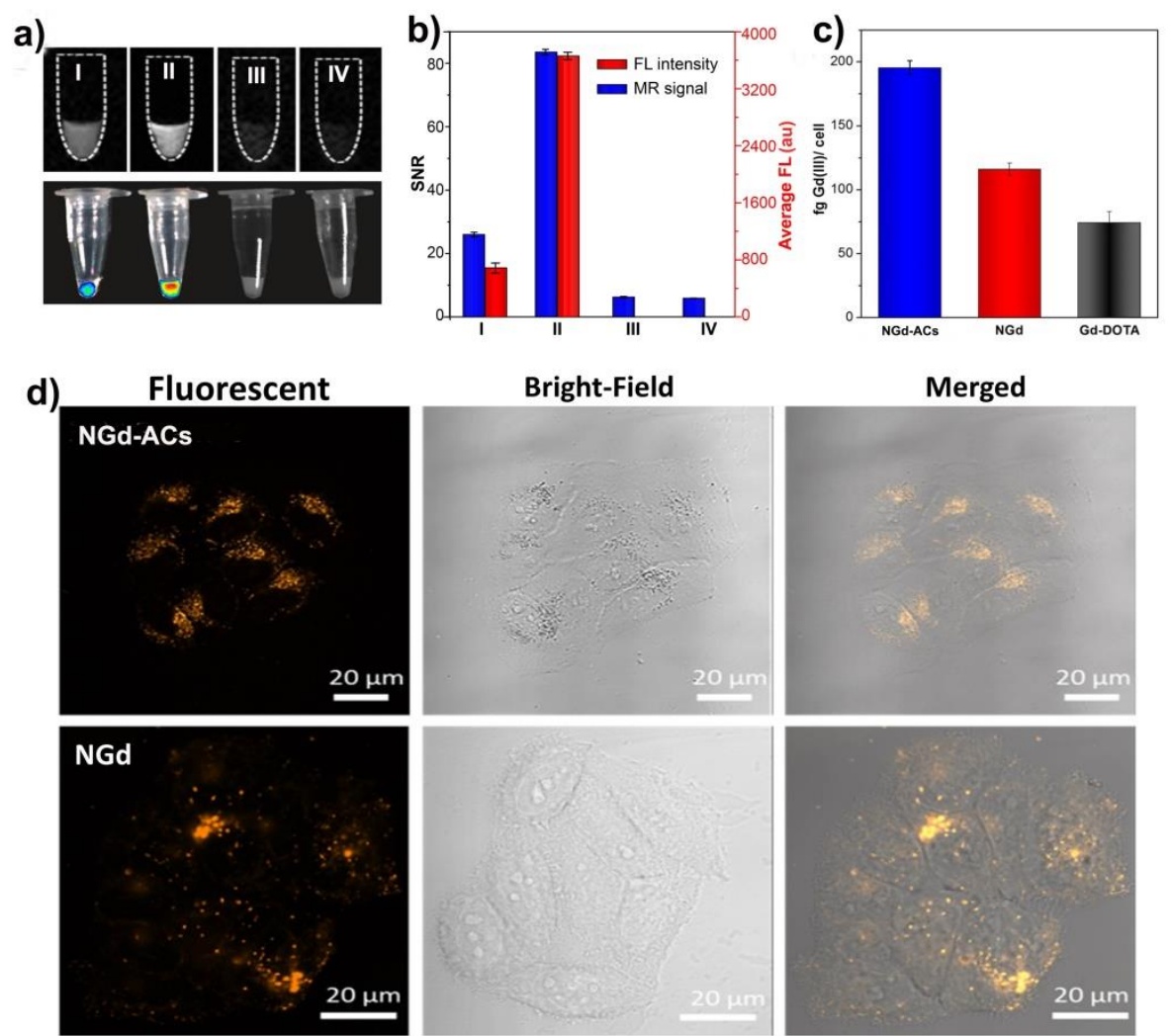

Merged

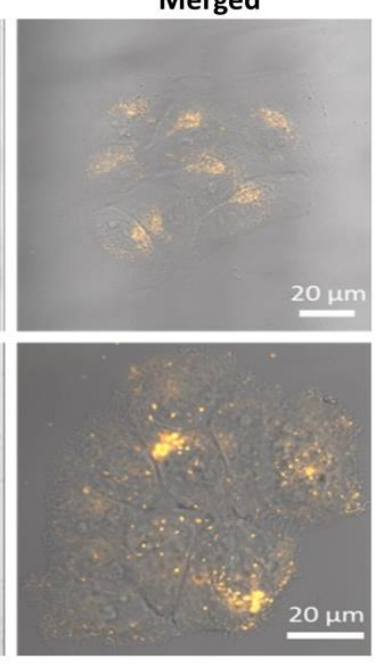

Figure 3. FLI and MRI in vitro. (a) $T_{1}$-weighted MRI (up) and fluorescence (down) images of cell pellets. (b) Comparison of signal to noise ratio (SNR) of $T_{1}$-weighted images ( $0.5 \mathrm{~T}$, blue) and the average FL intensity (red) of cell pellets in (a). I, II, III and IV refer to HeLa cells incubated with NGd, NGd-ACs, Gd-DOTA, and PBS, respectively. (c) Quantification of cellular uptake of NGd-ACs, NGd, and Gd-DOTA, respectively. (d) CLSM images of HeLa cells incubated with NGd-ACs and NGd. Scale bar: $20 \mu \mathrm{m},[\mathrm{NGd}-\mathrm{ACs}]=[\mathrm{NGd}]=10 \mu \mathrm{M}$. 

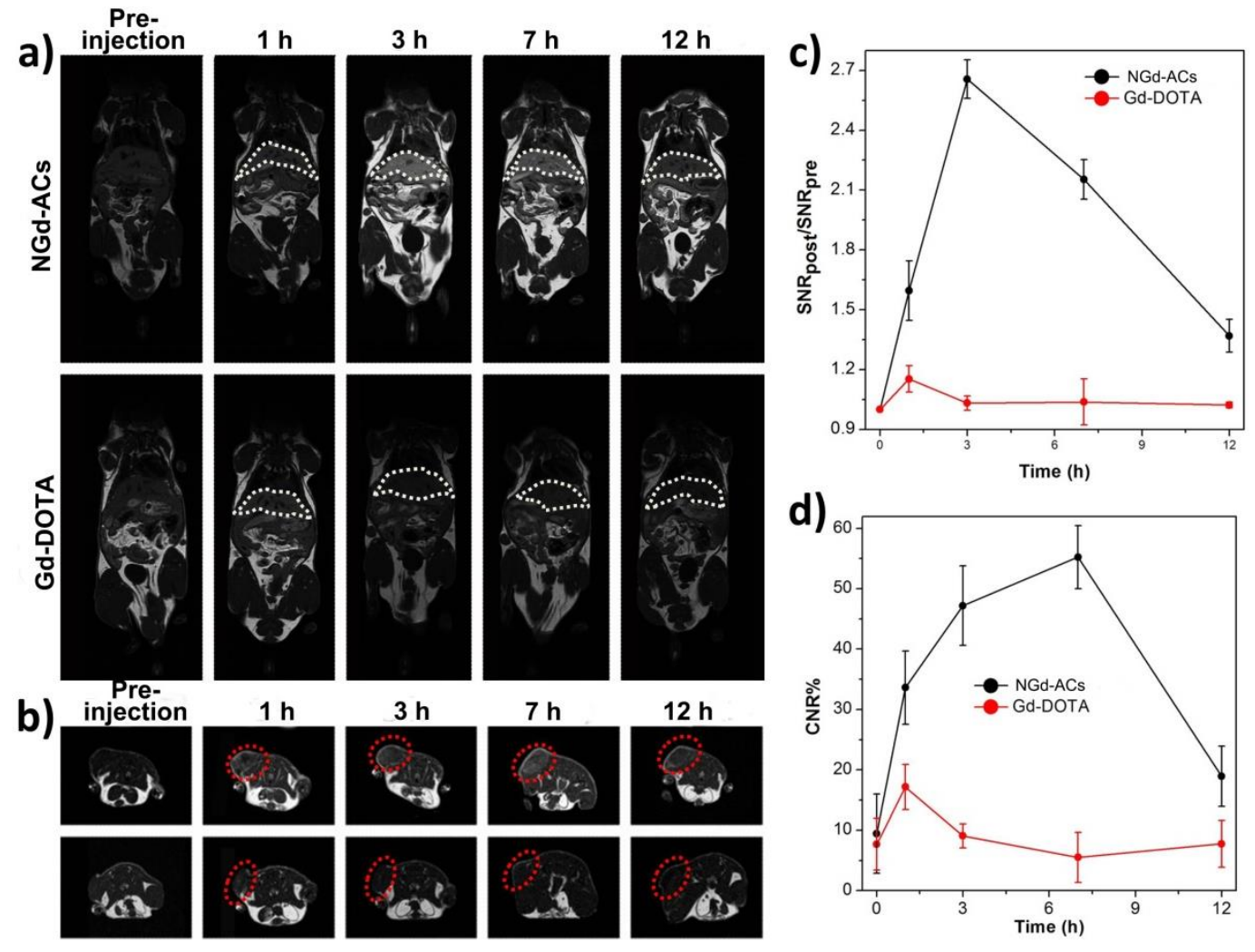

Figure 4. In vivo $T_{1}$-weighted MRI and analysis of mice after intravenous injection of NGdACs or Gd-DOTA. (a) MRI of liver with the dosage of $6 \mu \mathrm{M} \mathrm{Gd(III)/Kg} \mathrm{body} \mathrm{weight} \mathrm{before}$ and after intravenous injection at 1,3,7, and $12 \mathrm{~h}$, respectively. The white dotted lines refer to liver. (b) MRI of tumor before and after intravenous injection of NGd-ACs (up) and GdDOTA (down) at $1,3,7$, and $12 \mathrm{~h}$, respectively. The red dotted circles refer to tumor. (c) Quantitative analysis of signal changes (SNR ratio) in liver versus treating time. (d) Contrastto-noise ratios (CNR) of tumor and tissue contrast before and after injection of the probes at 1 , 3,7 , and $12 \mathrm{~h}$, respectively. $\mathrm{CNR}=\mid \mathrm{SNR}_{\text {tumor }}-\mathrm{SNR}_{\text {muscle }} / \mathrm{SNR}_{\text {muscle }}$. 


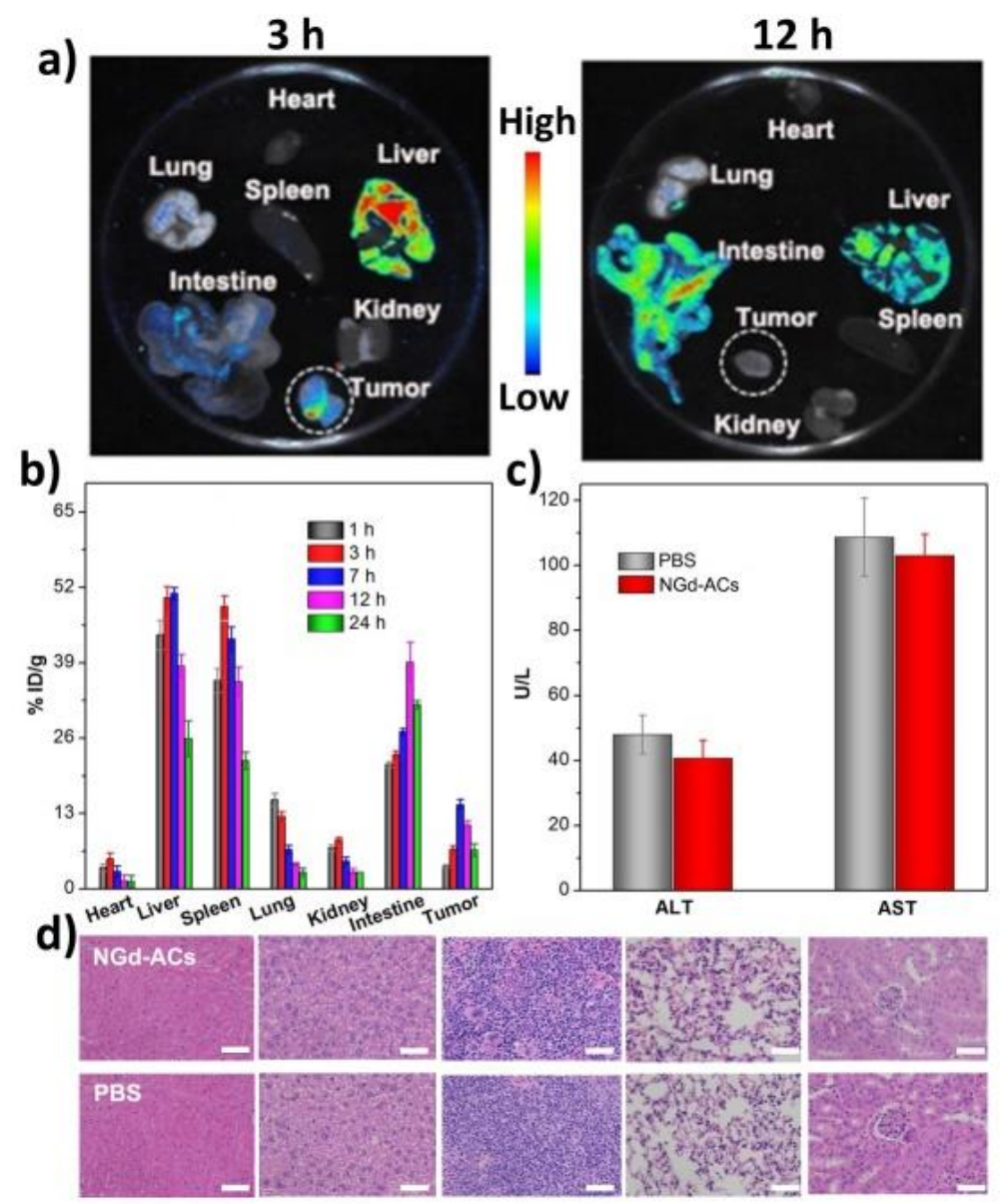

Figure 5. Biodistribution and biosafety assessment. (a) Ex vivo fluorescence images of main tissues (heart, liver, spleen, lung, kidney and intestine) and tumor from mice at 3 and $12 \mathrm{~h}$ post-injection of NGd-ACs. (b) Biodistribution of NGd-ACs at 1, 3, 7, and $12 \mathrm{~h}$ after injection in tumor-bearing mice evaluated by ICP-MS. (c) Serological test results of mice injected with NGd-ACs $(30 \mu \mathrm{mol} \mathrm{Gd(III)/kg} \mathrm{body} \mathrm{weight,} \mathrm{PBS} \mathrm{was} \mathrm{as} \mathrm{the} \mathrm{control).} \mathrm{(d)} \mathrm{H \& E} \mathrm{stained} \mathrm{tissue}$ sections from mice after intravenous injection of NGd-ACs (30 $\mu \mathrm{mol}$ Gd(III)/kg body weight) for 30 days, and PBS was used as a control. Scale bar: $50 \mu \mathrm{m}$ for all images. 


\section{The table of contents entry:}

A facile and straightforward strategy to synergistically enhance fluorescence quantum yield and $T_{1}$ relaxivity by albumin caged bimodal FLI/MRI probe of NGd-ACs is presented. This albumin cage organically integrates the mechanism of AIE and the prolonged rotation correlation time of gadolinium complex and diffusion correlation time of the surrounding water molecules, achieving an effect of $1+1>2$.

Keyword: albumin cage, fluorescence, magnetic resonance signal, aggregation-induced emission, synergetic enhancement

Lirong Wang, ${ }^{1}$ Qing Wan, ${ }^{1}$ Rongyuan Zhang, ${ }^{6}$ Bo Situ, ${ }^{3}$ Kaiyuan Ni, Jinhao Gao, ${ }^{2}$ Zhiming Wang, ${ }^{1, *}$ Anjun Qin, ${ }^{1, *}$ Ben Zhong Tang ${ }^{1,4}$

Synergetic Enhancement of Fluorescence and Magnetic Resonance Signals Assisted by Albumin Cage

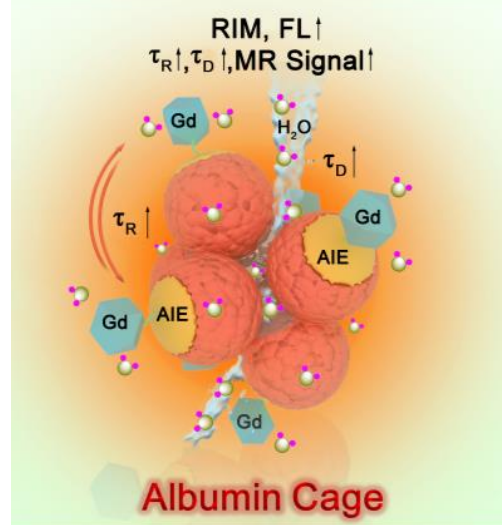




\section{Supporting Information}

\section{Synergetic Enhancement of Fluorescence and Magnetic Resonance Signals Assisted by Albumin Cage}

Lirong Wang, ${ }^{1}$ Qing Wan, ${ }^{1}$ Rongyuan Zhang, ${ }^{6}$ Bo Situ, ${ }^{3}$ Kaiyuan Ni, ${ }^{5}$ Jinhao Gao, ${ }^{2}$ Zhiming Wang, ${ }^{1, *}$ Anjun Qin, ${ }^{1, *}$ Ben Zhong Tang ${ }^{1,4}$

\section{Materials and Methods}

All chemicals, unless otherwise noted, were purchased from commercial sources and used without further purification. $\mathrm{GdCl}_{3}$, 1-ethyl-3-(3-dimethylamino-propyl) carbodiimide hydrochloide (EDC) and $N$-hydroxysulfo-succinimide (NHS) were purchased from Alfa Aesar. 1,4,7,10-Tetra-azacyclododecane (min. 98\% CYCLEN) was purchased from Strem Chemicals Inc.. Ultra-filtration units (Amicon Ultra $15 \mathrm{~mL}$ Filters with $30 \mathrm{kDa}$ nominal molecular weight cutoff) were purchased from Merck Millipore Corp. [4-(bis(4methoxyphenyl)amino)phenyl]boronic acid was obtained from Soochiral Chemical Science \& Technology Co., Ltd. 2,3-Dihydronaphthalene-2,3-diamine was purchased from Bide Pharmatech Ltd. Zinc chloride were obtained from Alfa Aesar. Sodium nitrite, potassium carbonate and sodium hydroxide were purchased from Aladdin Co., Ltd. Tetrakis(triphenylphosphine)palladium and 11-bromoundecanoic acid were obtained from Energy Co., Ltd.

${ }^{1} \mathrm{H}$ and ${ }^{13} \mathrm{C}$ NMR spectra were measured on a Bruker AV 500 spectrometer. High-resolution mass spectra (HR-MS) were recorded on a Acquity UPLC/XEVO G2-XS QTOF operating in MALDI-TOF mode. UV-vis absorption spectra were measured on a Shimadzu UV-2600 spectrophotometer. Photoluminescence spectra were recorded on a Horiba Fluoromax-4 spectrofluorometer. Photoluminescence (PL) quantum yields were measured using a Hamamatsu absolute PL quantum yield spectrometer C11347 Quantaurus_QY. Confocal laser scanning microscope (CLSM) characterization was conducted with a Zeiss LSM 710 
(Germany) confocal laser scanning biological microscope. The absorbance for MTT analysis was recorded on a Thermo Fisher microplate reader (USA) at a wavelength of $490 \mathrm{~nm}$. The element analysis of Gd was also carried out by inductively coupled plasma atomic emission spectroscopy (ICP-AES) or inductively coupled plasma mass spectroscopy (ICP-MS). The morphology and structure of NGd-BSA NPs with phosphotungstic acid staining (1 wt. \%) were characterized by transmission electron microscopy (TEM) using a JEOL JEM-2100 transmission electron microscope. The dynamic light scattering (DLS) measurements were performed on a Malvern Zetasizer nano ZS instrument. The $T_{1}$ relaxation time measurements were performed on 0.5 T NMI20-Analyst NMR Analyzing \& imaging system (Niumag Corporation, Shanghai, China), In vivo MR imaging was performed on a 1 T MRI System (Aspect M3 ${ }^{\mathrm{TM}}$, Israel). Ex vivo fluorescence imaging was carried out on Bruker FX Pro. 


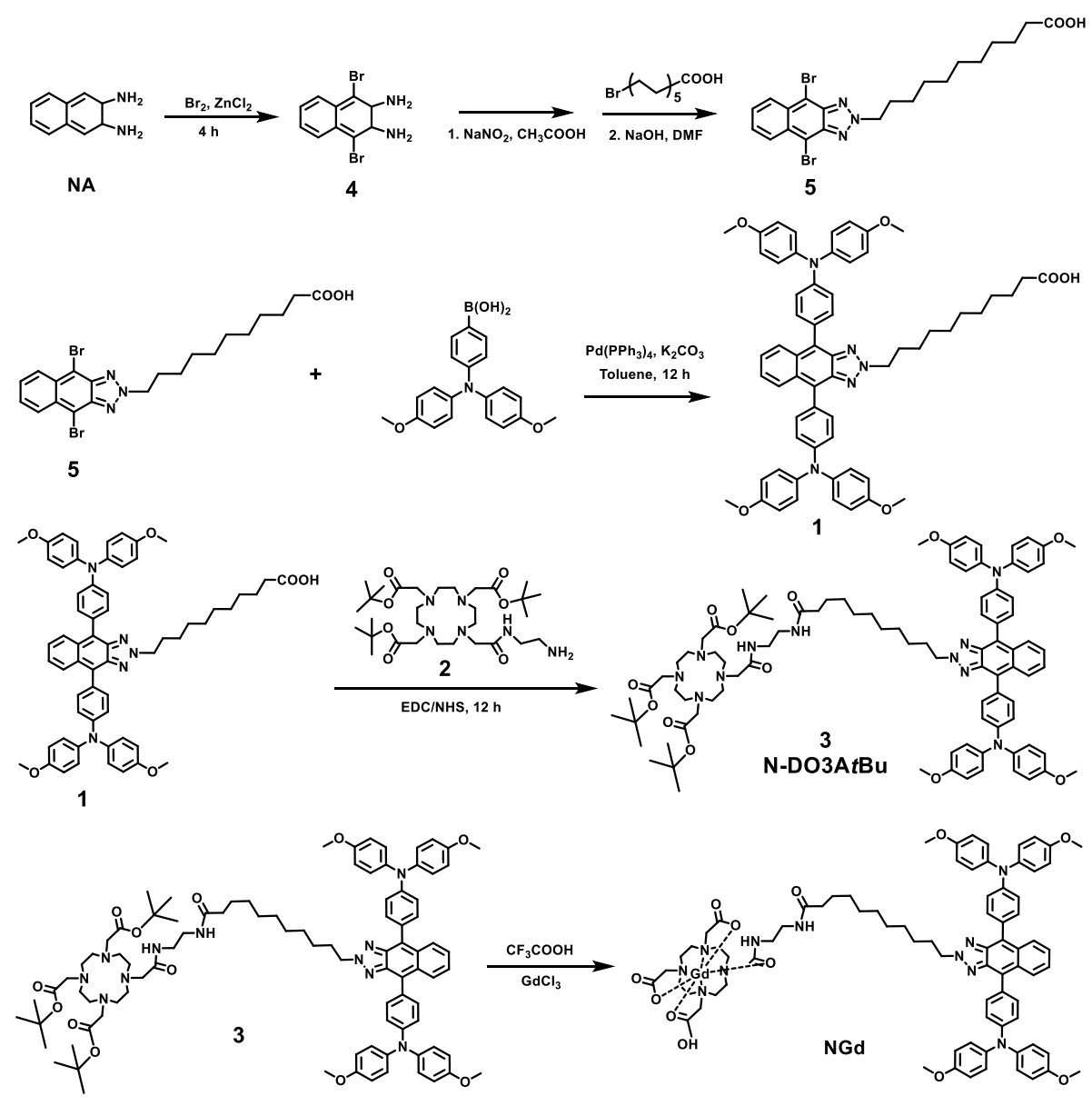

Scheme S1 Synthetic routes to NGd

\section{Synthesis of Intermediate 4}

The intermediate 4 was prepared by the bromination reaction according to previous literature. ${ }^{[1]}$ A mixture of $1.7 \mathrm{~mL}$ of bromine $(10.4 \mathrm{~g}, 43 \mathrm{mmol})$ and $50 \mathrm{~mL}$ of glacial acetic acid was gradually added into $70 \mathrm{~mL}$ of glacial acetic acid solution containing 2,3dihydronaphthalene-2,3-diamine (NA) $(2.5 \mathrm{~g}, 16 \mathrm{mmol})$, and then the solution was stirred at room temperature for $4 \mathrm{~h}$. The deionized water was added into solution, and the precipitate was filtered off and washed with glacial acetic acid and water. The brown powder was obtained after drying $(3.8 \mathrm{~g}, 81 \%)$.

\section{Synthesis of Intermediate 5}

The intermediate 5 was synthesized according to previous literature. ${ }^{[2]} 15 \mathrm{~mL}$ of $\mathrm{NaNO}_{2}$ aqueous solution $(3.0 \mathrm{~g}, 33.0 \mathrm{mmol})$ was added dropwise to $30 \mathrm{~mL}$ of glacial acetic acid containing compound $4(3.16 \mathrm{~g}, 10 \mathrm{mmol})$. After vigorous stirring for $30 \mathrm{~min}$ at room 
temperature, the precipitate was filtered off and washed with water to obtain brown powder. The crude product was directly used without further purification. The obtained brown powder (2.5 g, $7.6 \mathrm{mmol})$ was dissolved in dry DMF $(30 \mathrm{~mL})$ and $\mathrm{NaOH}(11.4 \mathrm{mmol}, 456 \mathrm{mg})$ was added with vigorous stirring within $1 \mathrm{~h}$. 11-Bromoundecanoic acid (4.1 g, $15.2 \mathrm{mmol})$ in DMF (20 mL) was added dropwise and the solution was stirred at room temperature for $24 \mathrm{~h}$. Afterward, the solution was adjusted to faintly acid by dilute hydrochloric acid. Then, the solution was extracted with DCM/water to remove DMF, the organic layer was dried over anhydride $\mathrm{MgSO}_{4}$. After filtration and solvent evaporation, the crude product was purified by column chromatography using ethyl acetate/petroleum ether mixture as eluent to afford orange powder $(2.8 \mathrm{~g}, 73 \%) .{ }^{1} \mathrm{H}$ NMR (DMSO- $\left.d_{6}, 500 \mathrm{MHz}\right): \delta 11.88(\mathrm{~s}, 1 \mathrm{H}), 8.33(\mathrm{~m}, 2 \mathrm{H})$, $7.70(\mathrm{~m}, 2 \mathrm{H}), 4.91(\mathrm{t}, 2 \mathrm{H}), 2.17(\mathrm{~m}, 4 \mathrm{H}), 1.32-1.20(\mathrm{~m}, 14 \mathrm{H}) .{ }^{13} \mathrm{C}$ NMR (DMSO- $d_{6}, 500$ MHz): $\delta 174.9142 .4,130.9,128.1,126.9,108.5,58.1,34.1,29.8,29.2,29.1,28.9,28.7,26.3$, 24.9. HR-MS calculated for $\mathrm{C}_{21} \mathrm{H}_{26} \mathrm{Br}_{2} \mathrm{~N}_{3} \mathrm{O}_{2}[\mathrm{M}+\mathrm{H}]^{+} m / z: 510.0392$, found: 510.0387 .

\section{Synthesis of Compound 1}

The Compound 1 was synthesized by Suzuki coupling reaction. The mixture of [4-(bis(4methoxyphenyl)amino)phenyl]boronic acid (2.1 g, $6 \mathrm{mmol})$, intermediate 5 (1.53 g, $3 \mathrm{mmol})$ and $\mathrm{Pd}\left(\mathrm{PPh}_{3}\right)_{4}(116 \mathrm{mg}, 0.1 \mathrm{mmol})$ were dissolved in $20 \mathrm{~mL}$ toluene and $12 \mathrm{~mL} \mathrm{~K} \mathrm{CO}_{3}(2 \mathrm{M})$ aqueous solution. Then, the mixture was refluxed under nitrogen for $24 \mathrm{~h}$. After solvent evaporation, the crude product was purified by column chromatography using ethyl acetate/petroleum ether mixture as eluent to afford orange powder $(1.7 \mathrm{~g}, 89 \%) .{ }^{1} \mathrm{H}$ NMR (DMSO-d $\left.d_{6}, 500 \mathrm{MHz}\right): \delta 8.04(\mathrm{~d}, 2 \mathrm{H}), 7.45(\mathrm{~d}, 2 \mathrm{H}), 7.35(\mathrm{~d}, 2 \mathrm{H}), 7.19(\mathrm{~d}, 2 \mathrm{H}), 6.96(\mathrm{~d}, 2 \mathrm{H})$, $8.92(\mathrm{~d}, 2 \mathrm{H}), 4.80(\mathrm{t}, 2 \mathrm{H}), 3.76(\mathrm{~s}, 12 \mathrm{H}), 1.98(\mathrm{t}, 2 \mathrm{H}), 1.87(\mathrm{t}, 2 \mathrm{H}), 1.26-1.15(\mathrm{~m}, 14 \mathrm{H}) .{ }^{13} \mathrm{C}$ NMR (DMSO- $d_{6}, 500$ MHz): $\delta 174.9,156.5,148.3,141.8,140.3,133.6,132.8,132.5,132.3$, $132.0,131.9,129.2,127.8,118.3,115.5,55.6,34.1,30.1,29.2,28.9,26.4,24.9$. HR-MS calculated for $\mathrm{C}_{61} \mathrm{H}_{62} \mathrm{~N}_{5} \mathrm{O}_{6}[\mathrm{M}+\mathrm{H}]^{+} m / z: 960.4700$, found: 960.4717 . 
Compound 1 (100 mg, $0.1 \mathrm{mmol}$ ), 1-(3-dimethylaminopropyl)-3-ethylcarbodiimide hydrochloride (EDC) (28.7 mg, $0.15 \mathrm{mmol}), N$-hydroxysuccinimide (NHS) (17.3 mg, 0.15 mmol) were dissolved in dry DMF $(10 \mathrm{~mL})$ and stirring at room temperature for $1 \mathrm{~h}$. The DMF solution (2 mL) of compound 2 [tri-tert-butyl 2,2',2'-(10-(2-((2-aminoethyl) amino)-2oxoethyl)-1,4,7,10-tertraazacyclododecane-1,4,7-triyl)triacetate, $67.5 \mathrm{mg}, 0.11 \mathrm{mmol}]$, which was synthesized according to the previous procedures, ${ }^{[3]}$ was added and the mixture was stirred at $50{ }^{\circ} \mathrm{C}$ for $10 \mathrm{~h}$. Afterward, the solution was extracted with DCM/water, the organic layer was dried over anhydride $\mathrm{MgSO}_{4}$. After filtration and solvent evaporation, the crude product was purified by column chromatography using methanol/DCM mixture as eluent to afford light-yellow powder compound $3(99.5 \mathrm{mg}, 64 \%) .{ }^{1} \mathrm{H} \mathrm{NMR}\left(500 \mathrm{MHz}, \mathrm{CD}_{2} \mathrm{Cl}_{2}\right)$ : $\delta$ $8.14(\mathrm{dd}, 2 \mathrm{H}), 7.49(\mathrm{~d}, 4 \mathrm{H}), 7.30(\mathrm{dd}, 2 \mathrm{H}), 7.21(\mathrm{~d}, 8 \mathrm{H}), 7.07(\mathrm{~d}, 4 \mathrm{H}), 6.91(\mathrm{~d}, 8 \mathrm{H}), 4.80(\mathrm{t}$, 2H), $3.81(\mathrm{~s}, 12 \mathrm{H}), 3.34-3.19(\mathrm{~m}, 4 \mathrm{H}), 2.83-2.08(\mathrm{~m}, 26 \mathrm{H}), 1.84(\mathrm{~s}, 2 \mathrm{H}), 1.71-0.96(\mathrm{~m}$, 41H). ${ }^{13} \mathrm{C}$ NMR $\left(500 \mathrm{MHz}, \mathrm{CD}_{2} \mathrm{Cl}_{2}\right): \delta 177.82,177.57,175.02,172.94,170.64,157.45$, $149.82,143.82,141.63,133.36,131.33,129.18,128.42,128.04,127.87,127.72,125.63$, $121.71,120.37,116.12,91.90,56.78,54.75,45.25,43.64,39.49,37.49,36.54,35.73,33.21$, $32.45,31.67,31.01,30.96,30.86,30.70,30.59,30.44,30.36,29.98,29.24,27.95,26.24$, 24.01, 15.93, 15.20. HR-MS calculated for $\mathrm{C}_{91} \mathrm{H}_{118} \mathrm{~N}_{11} \mathrm{O}_{12}[\mathrm{M}+\mathrm{H}]^{+} m / z$ : 1556.8961, found: 1556.8981.

\section{Synthesis of NGd}

Compound 3 (90 mg, $0.06 \mathrm{mmol}$ ) was dissolved in $3 \mathrm{~mL}$ trifluoroacetate acid (TFA) at room temperature for deprotection. After stirring for $6 \mathrm{~h}$, excess TFA was removed by rotary evaporation. And then, the resultant brown oily product was dissolved in methanol, and the $\mathrm{pH}$ of the solution was adjusted to 5 6 with dilute sodium hydroxide solution. Anhydrous gadolinium chloride (24 mg, $0.09 \mathrm{mmol}$ ) was dissolved into $3 \mathrm{~mL}$ methanol, and added dropwise into the above brown solution. After reaction overnight at $60{ }^{\circ} \mathrm{C}$, the methanol was removed by evaporation. The crude product was re-dissolved in DCM, and the excess 
gadolinium ions were washed off with water to obtain the brown solid product $(92.5 \mathrm{mg}$, 90\%). HR-MS (m/z) calculated for $\mathrm{C}_{79} \mathrm{H}_{91} \mathrm{GdN}_{11} \mathrm{O}_{12}[\mathrm{M}+\mathrm{H}]^{+} m / z:$ 1542.6011, found: 1542.6017.

\section{Binding constant of NGd to BSA}

The binding constant of NGd to BSA was assessed by proton relaxation enhancement (PRE), which is commonly used to determine affinity constant of $\mathrm{Gd}(\mathrm{III})$ complexes to serum albumin. ${ }^{[4]}$ The water proton relaxation time was measured with the increasing concentrations of BSA in PBS buffer while the concentration of NGd keeps unchanged at $10 \mu \mathrm{M}$. The binding constant was calculated according to the following equations 1 to 3 .

$$
\begin{aligned}
\mathrm{NGd}+\mathrm{BSA} \leftrightarrows \mathrm{NGd}-\mathrm{BSA} \\
\mathrm{K}_{\mathrm{D}}=\frac{[\mathrm{NGd}][\mathrm{BSA}]}{[\mathrm{NGd}-\mathrm{BSA}]} \\
\varepsilon^{*}=\frac{\left(1 / \mathrm{T}_{1}\right)_{\mathrm{NGd}-\mathrm{BSA}}-\left(1 / \mathrm{T}_{1}\right)_{\mathrm{BSA}}}{\left(1 / \mathrm{T}_{1}\right)_{\mathrm{NGd}-\mathrm{PBS}}-\left(1 / \mathrm{T}_{1}\right)_{\mathrm{PBS}}} \\
\varepsilon^{*}=\frac{\varepsilon_{\mathrm{max}}[\mathrm{BSA}]_{\mathrm{t}}+\mathrm{K}_{\mathrm{D}}}{\mathrm{K}_{\mathrm{D}}+[\mathrm{BSA}]_{\mathrm{t}}}
\end{aligned}
$$

Where, $K_{\mathrm{D}}$ is the binding dissociation constant; [NGd-BSA] is the concentration of NGd-BSA complex; [NGd] is the concentration of free NGd in solution; [BSA] is the concentration of free $\mathrm{BSA}$ in solution; $[\mathrm{BSA}]_{\mathrm{t}}$ is the total concentration of $\mathrm{BSA} ; \varepsilon^{*}$ is the enhancement factor; $\varepsilon_{\max }$ is the maximum value that the enhancement factor can reach extrapolated to the scenario where all the contrast agent molecular are almost bound to BSA; $\left(1 / \mathrm{T}_{1}\right)_{\mathrm{NGd}-\mathrm{BSA}},\left(1 / \mathrm{T}_{1}\right)_{\mathrm{BSA}}$, $\left(1 / \mathrm{T}_{1}\right)_{\mathrm{NGd}-\mathrm{PBS}},\left(1 / \mathrm{T}_{1}\right)_{\mathrm{PBS}}$ are the relaxation rates of water protons in NGd plus BSA, BSA alone, NGd plus PBS, and PBS alone, respectively.

\section{Synthesis of NGd-ACs}

NGd molecules were dissolved in DMSO at a Gd(III) concentration of $16.7 \mathrm{mM}$ and kept in $4{ }^{\circ} \mathrm{C}$ refrigerator. NGd-ACs were synthesized by desolvation followed by glutaraldehyde coupling. ${ }^{[3 \mathrm{a}, 5]} 181.4 \mu \mathrm{L}$ of NGd was diluted to $20 \mathrm{~mL}$ THF and dropwise added into $50 \mathrm{~mL}$ of 
BSA ( 20 mg/mL) aqueous solution. The molar ratio of BSA/NGd is 1:2. The resultant mixture was stirred for $30 \mathrm{~min}$ at room temperature. Under a stirring at $400 \mathrm{rpm}$, additional THF was dropwise added to the above mixture until the solution became slightly turbid. Then $100 \mu \mathrm{L}$ of $2.5 \%$ glutaraldehyde aqueous solution was added, and the reaction was covered with aluminum foils and stirring at room temperature. After reaction for $4 \mathrm{~h}$, the mixture was concentrated in vacuum to remove organic solutions at room temperature. And the NGd-ACs were purified three times by ultrafiltration with Amicon centrifuge tubes (MWCO $=30 \mathrm{kDa}$ ) and re-dispersion in DI-water under sonication. The Gd(III) concentration was measured by inductively coupled plasma atomic emission spectroscopy (ICP-AES). The encapsulation efficiency (EE) of NGd was calculated according to the following equation 4.

$$
\mathrm{EE}=\frac{\text { Mass of encapsulated NGd }}{\text { Total mass of added NGd }} \times 100 \%
$$

\section{$T_{1}$ Relaxivity and MR Phantom Study}

The $T_{1}$ relaxation times of $\mathrm{NGd}-\mathrm{ACs}$, NGd in BSA aqueous solution (molar ratio of BSA/NGd = 1:2), NGd in water, and Gd-DOTA were measured at $0.5 \mathrm{~T}$ MRI scanner with the $\mathrm{Gd}(\mathrm{III})$ concentration of $0.40,0.20,0.10,0.05$, and $0.025 \mathrm{mM}$, respectively. Then, the values of $r_{1}$ were calculated through the curve fitting of relaxation rates $\left(\mathrm{s}^{-1}\right)$ versus the $\mathrm{Gd}(\mathrm{III})$ concentration $(\mathrm{mM})$. Meanwhile, $T_{1}$-weighted phantom images were carried out with TR/TE $=100 / 0.004 \mathrm{~ms}$.

\section{Cell culture}

HeLa cells were cultured in Dulbecco's modified eagle medium (DMEM) containing 10\% fetal bovine serum at $37{ }^{\circ} \mathrm{C}$ in a humidified environment containing $5 \% \mathrm{CO}_{2}$.

\section{Confocal Microscopy}

HeLa cells were seeded at a density of $5 \times 10^{4}$ cells per plate on a $35 \mathrm{~mm}$ dish. Cells were incubated with the same Gd(III) concentration $(10 \mu \mathrm{M})$ of NGd-ACs and NGd for $24 \mathrm{~h}$. Cells were washed twice with PBS and fresh media was added. Cells were imaged using a Zeiss LSM 710 confocal laser scanning with the excitation of $405 \mathrm{~nm}$ and emission of 500-600 nm. 


\section{Cell viability}

The cytotoxicity of NGd-ACs and NGd were assessed by the 3-(4, 5-dimethylthiazol-2-y1)2, 5-diphenyltetrazolium bromide (MTT) method. HeLa cells were firstly seeded into a 96well plate at a density of $1 \times 10^{4}$ cells per well in DMEM, and incubated for $24 \mathrm{~h}$. Then the media were replaced by the different Gd(III) concentrations of NGd-ACs and NGd $(0.78,1.56$, $3.13,6.25,12.5,25,50,100 \mu \mathrm{M})$ and cells were incubated for another $24 \mathrm{~h}$. After incubation, the culture media were removed and each well was filled with $100 \mu \mathrm{L}$ of new culture media containing MTT $(0.5 \mathrm{mg} / \mathrm{mL})$ and incubation for additional $4 \mathrm{~h}$. Then the media was discarded and each well was added with another $100 \mu \mathrm{L}$ DMSO. The OD490 value (Abs.) of each well was measured by microplate reader immediately. Cell viability was expressed by the ratio of OD490 values of the cells incubated with NGd-ACs or NGd suspension to that of the cells incubated with culture medium only.

\section{Cell MR/FL imaging and cell uptake}

HeLa cells at a density of $5 \times 10^{6}$ per dish were seeded into four tissue culture dishes respectively. After incubation for $24 \mathrm{~h}$, these media were replaced by the same Gd(III) concentration $(100 \mu \mathrm{M})$ of media containing NGd-ACs, NGd, and Gd-DOTA respectively, PBS was used as blank group. The cells of four groups were washed with PBS three times and harvested into $200 \mu \mathrm{L}$ microcentrifuge tube, respectively. $T_{1}$-weighted phantom images of cells were obtained on a 0.5 T NMR120-Analyst NMR system with parameters: TR $=100 \mathrm{~ms}$ and $\mathrm{TE}=5.3 \mathrm{~ms}$. The regions of interest were measured by ImageJ. After that, fluorescence images of these samples were collected using BIO-RAD ChemiDoc ${ }^{\mathrm{TM}}$ MP gel imaging system with white light excitation.

After imaging testing, the collected cells were nitrified by $70 \%$ (wt/vol) $\mathrm{HNO}_{3}$ at $100{ }^{\circ} \mathrm{C}$ overnight. The digested solutions were collected and diluted to $10 \mathrm{~mL}$. The $\mathrm{Gd}$ content in each sample was analyzed by ICP-MS.

\section{In vivo MR Imaging}


All animal studies were approved by Institutional Animal Care and Use Committee (IACUC) of South China University of Technology.

MR imaging of BALB/c tumor bearing mice (6 weeks) were carried out on a $1 \mathrm{~T}$ MR scanner (Aspect M3 ${ }^{\mathrm{TM}}$, Israel). NGd-ACs were intravenously injected into mice with a dose of $6 \mu \mathrm{mol} \mathrm{Gd} / \mathrm{kg}$ body weight. Gd-DOTA as clinically used MRI contrast agent was employed as control. $T_{1}$-weighted images were acquired at different time points after injection. All the images were obtained using spin-echo sequence (SE) with parameters as follows: TR/TE = $330 / 12.5 \mathrm{~ms}$, slice thickness $=1 \mathrm{~mm}, \mathrm{FOV}=30 \times 90$

\section{Ex vivo FL imaging}

$\mathrm{BALB} / \mathrm{c}$ tumor bearing mice were intravenously injected with $6 \mu \mathrm{mol} \mathrm{Gd}(\mathrm{III}) / \mathrm{kg}$ body weight. Organs (heart, liver, spleen, lung, kidney, intestine) and tumors were harvested after injection for 3 and $12 \mathrm{~h}$. Organs were imaged on the Bruker FX Pro with $\lambda_{\mathrm{ex}}=410 \mathrm{~nm}$ and $\lambda_{\mathrm{em}}$ $=535 \mathrm{~nm}$.

\section{Biodistribution in Tumor-Bearing Mice}

$\mathrm{BALB} / \mathrm{c}$ tumor bearing mice were injected with NGd-ACs and NGd at the same dose of 30 $\mu \mathrm{mol} \mathrm{Gd(III)/kg} \mathrm{body} \mathrm{weight} \mathrm{and} \mathrm{were} \mathrm{sacrificed} \mathrm{after} \mathrm{injection} \mathrm{for} 1,3,7$, and $24 \mathrm{~h}(n=$ 4/group). Tumors and major organs (heart, liver, spleen, lung, kidney, intestine) were collected and weighed, and chemically digested using the method of microwave shock with $\mathrm{HNO}_{3} / \mathrm{H}_{2} \mathrm{O}_{2}(3: 1 \mathrm{v} / \mathrm{v})$ at $100{ }^{\circ} \mathrm{C}$. Each digested sample was diluted to a certain volume with $2 \% \mathrm{HNO}_{3}$ and filtered through a $0.22 \mu \mathrm{m}$ pore filter membrane. The $\mathrm{Gd}(\mathrm{III})$ concentrations in these samples were measured using ICP-MS. The final Gd(III) concentrations were expressed as the percentage of the injected dose per gram tissue $(\% \mathrm{ID} / \mathrm{g})$.

\section{Serological test and Histological Examination}

$\mathrm{BALB} / \mathrm{c}$ mice were intravenous injected with NGd-ACs at the dosage of $30 \mu \mathrm{mol}$ $\mathrm{Gd}(\mathrm{III}) / \mathrm{kg}$ mouse body weight as the experimental group $(n=4)$. The mice of the control group $(n=4)$ were injected with the same volume of PBS solution. After 30 days, blood was 
collected by heart to evaluate the liver function, including alanine aminotransferase (ALT) and aspartate aminotransferase (AST). In addition, the main organs including heart, liver, spleen, lung, and kidneys were collected, sectioned and stained with hematoxylin and eosin. The histological sections were analyzed for the study of in vivo toxicity. 


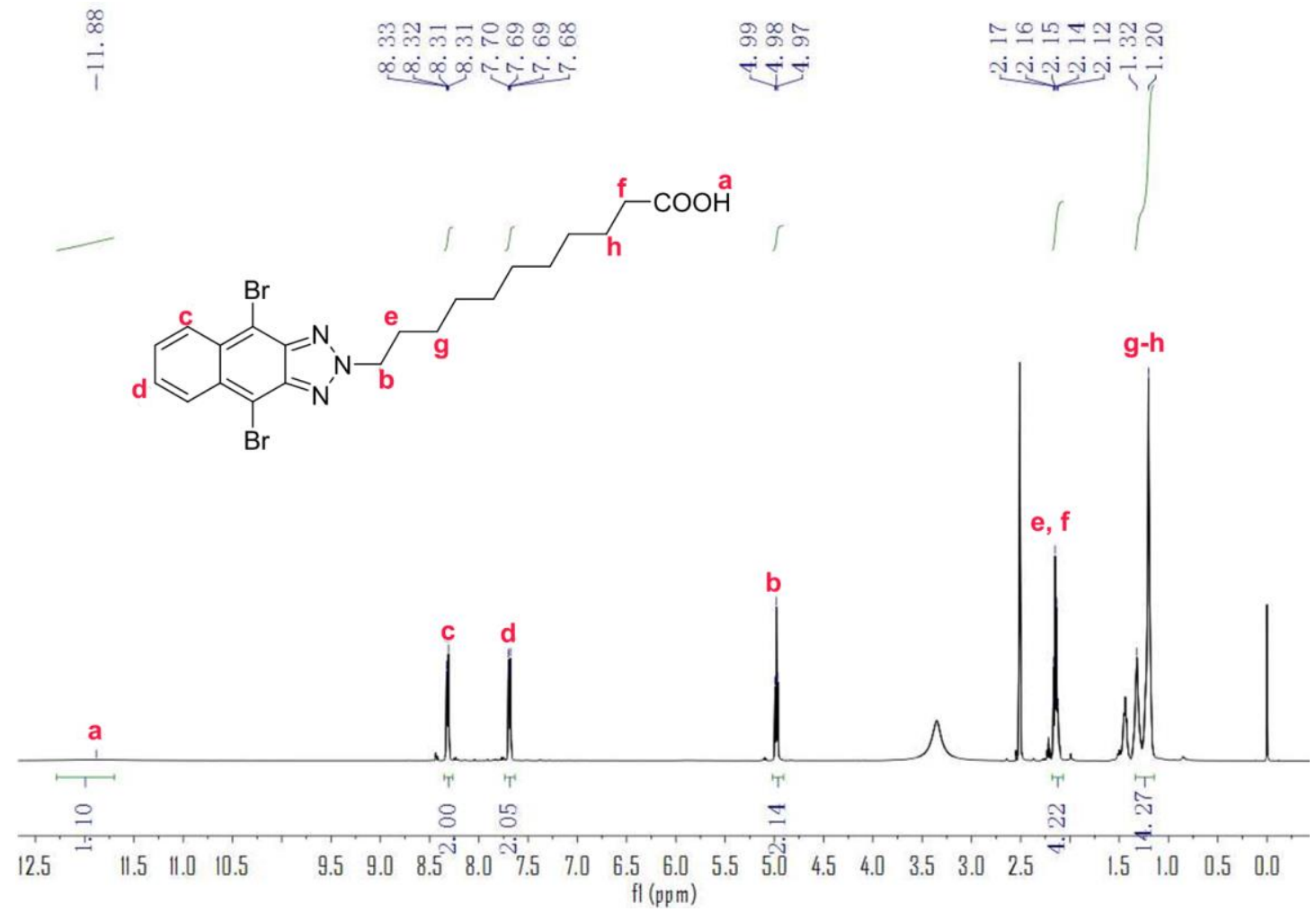

Figure S1. ${ }^{1} \mathrm{H}$ NMR of Intermediate 5

热焉

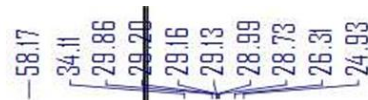
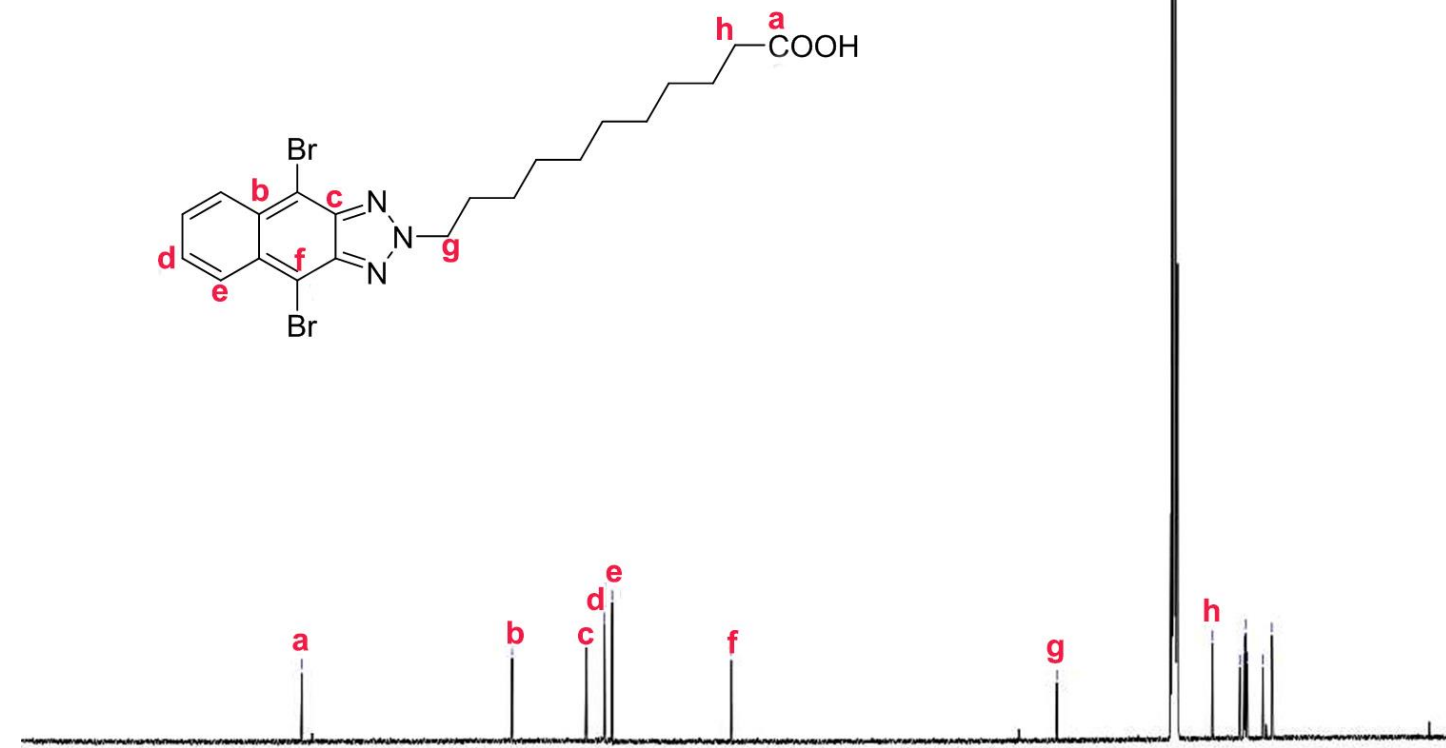

$\begin{array}{llllllllllllllllllllll}210 & 190 & 180 & 170 & 160 & 150 & 140 & 130 & 120 & 110 & 100 & 90 & 80 & 70 & 60 & 50 & 40 & 30 & 20 & 10 & 0\end{array}$

Figure S2. ${ }^{13} \mathrm{C}$ NMR of Intermediate 5 


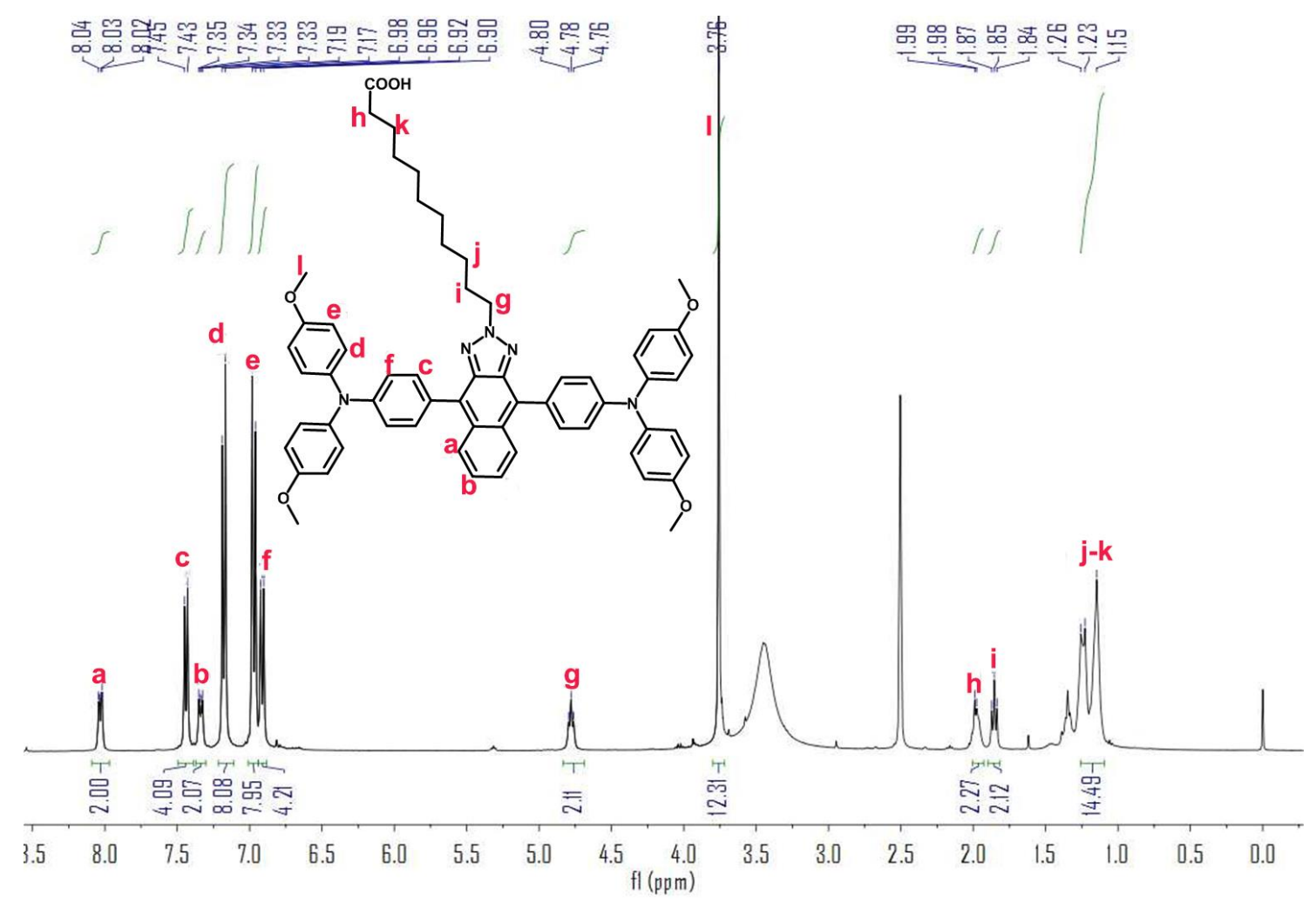

Figure S3. ${ }^{1} \mathrm{H}$ NMR of Compound 1 


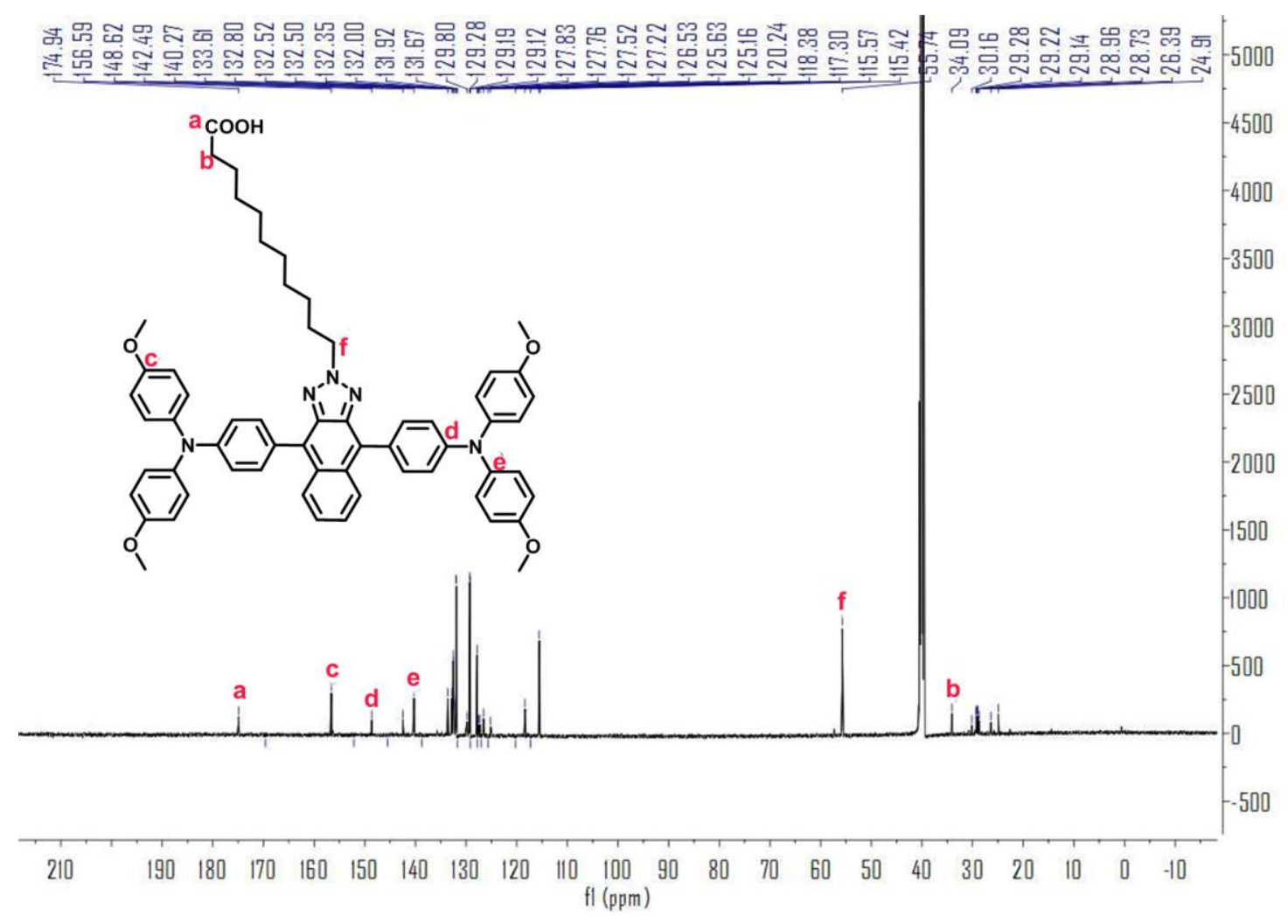

Figure S4. ${ }^{13} \mathrm{C}$ NMR of Compound 1 


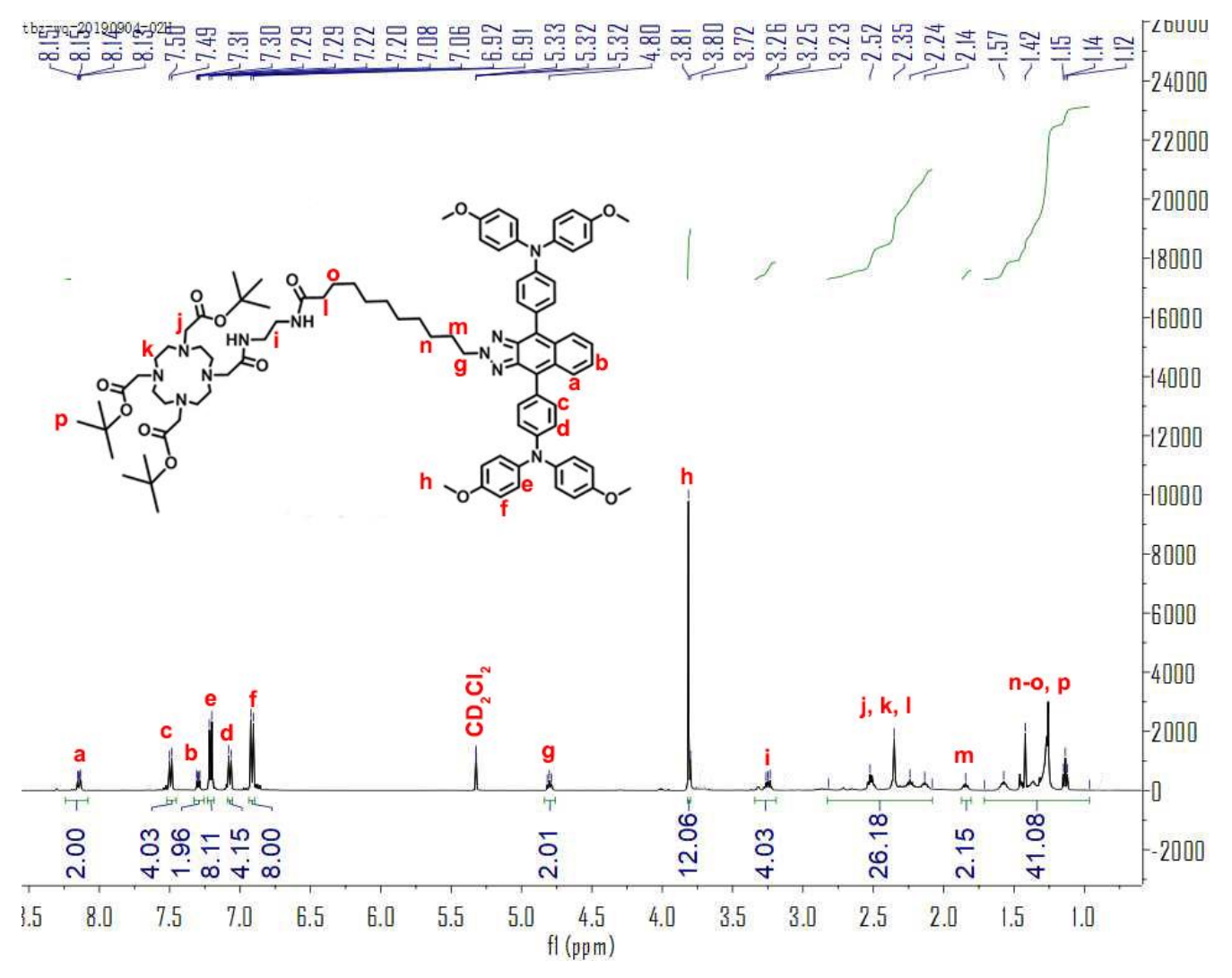

Figure S5. ${ }^{1} \mathrm{H}$ NMR of Compound $3(\mathrm{~N}-\mathrm{DO} 3 \mathrm{~A} t \mathrm{Bu})$

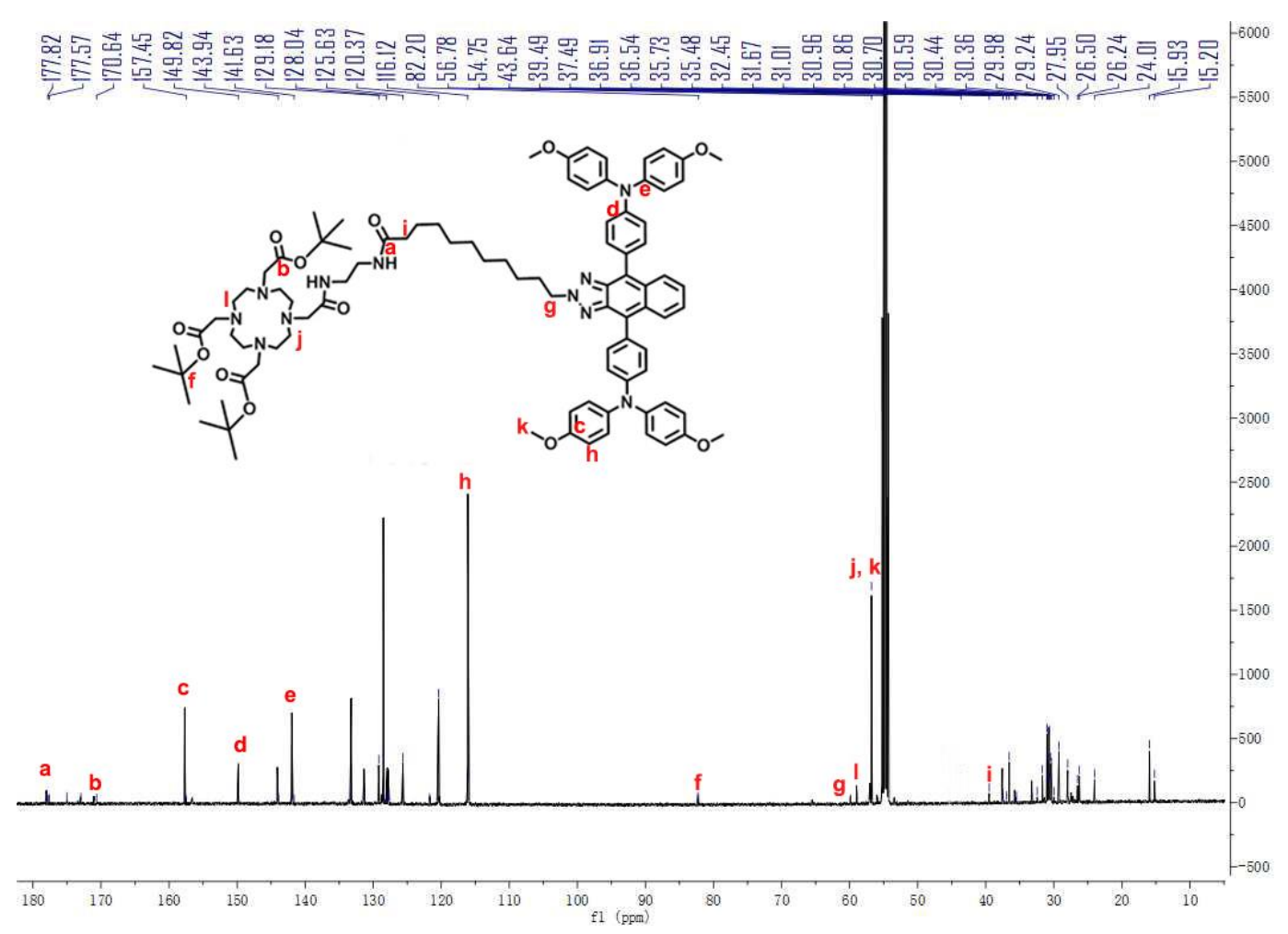

Figure S6. ${ }^{13} \mathrm{C}$ NMR of Compound $3(\mathrm{~N}-\mathrm{DO} 3 \mathrm{~A} t \mathrm{Bu})$ 


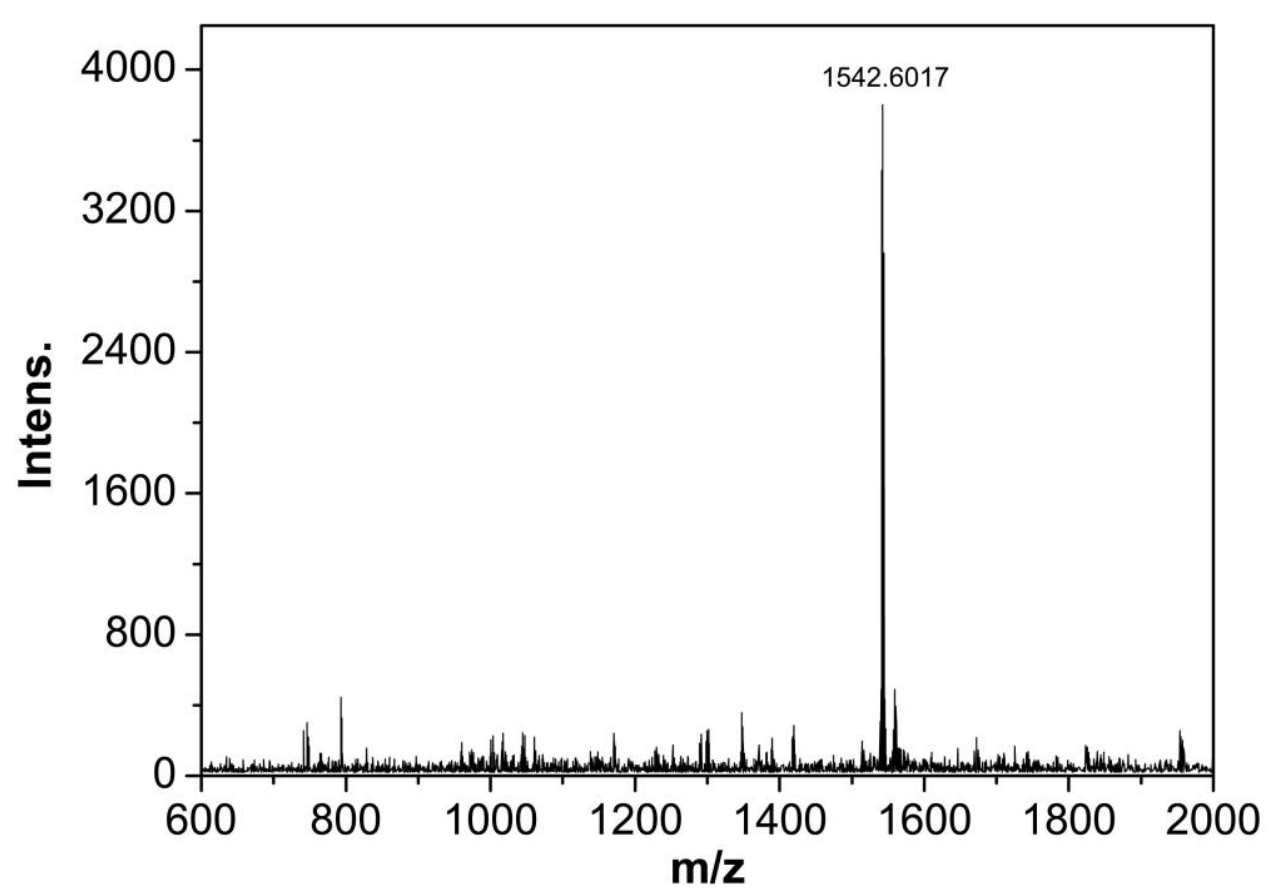

Figure S7. HR-MS of NGd 


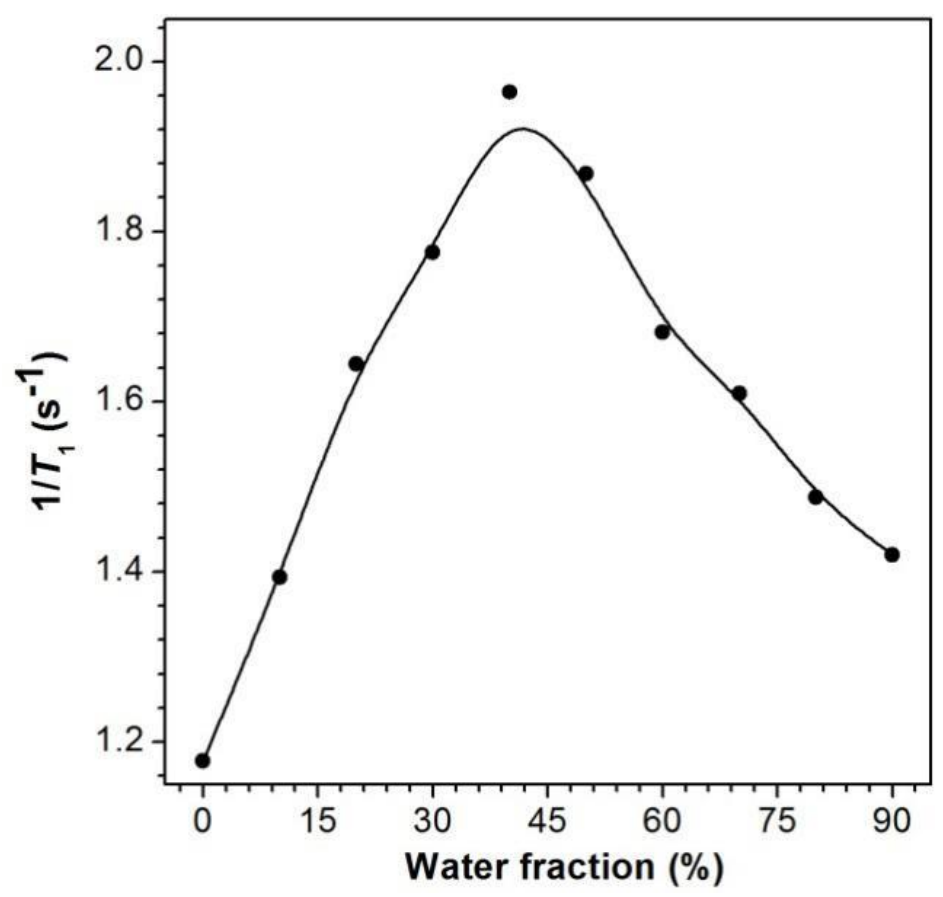

Figure S8. The relaxivities of NGd (0.1 mM [Gd]) in mixtures of DMSO/water with different water fraction $\left(f_{\mathrm{w}}\right)$.
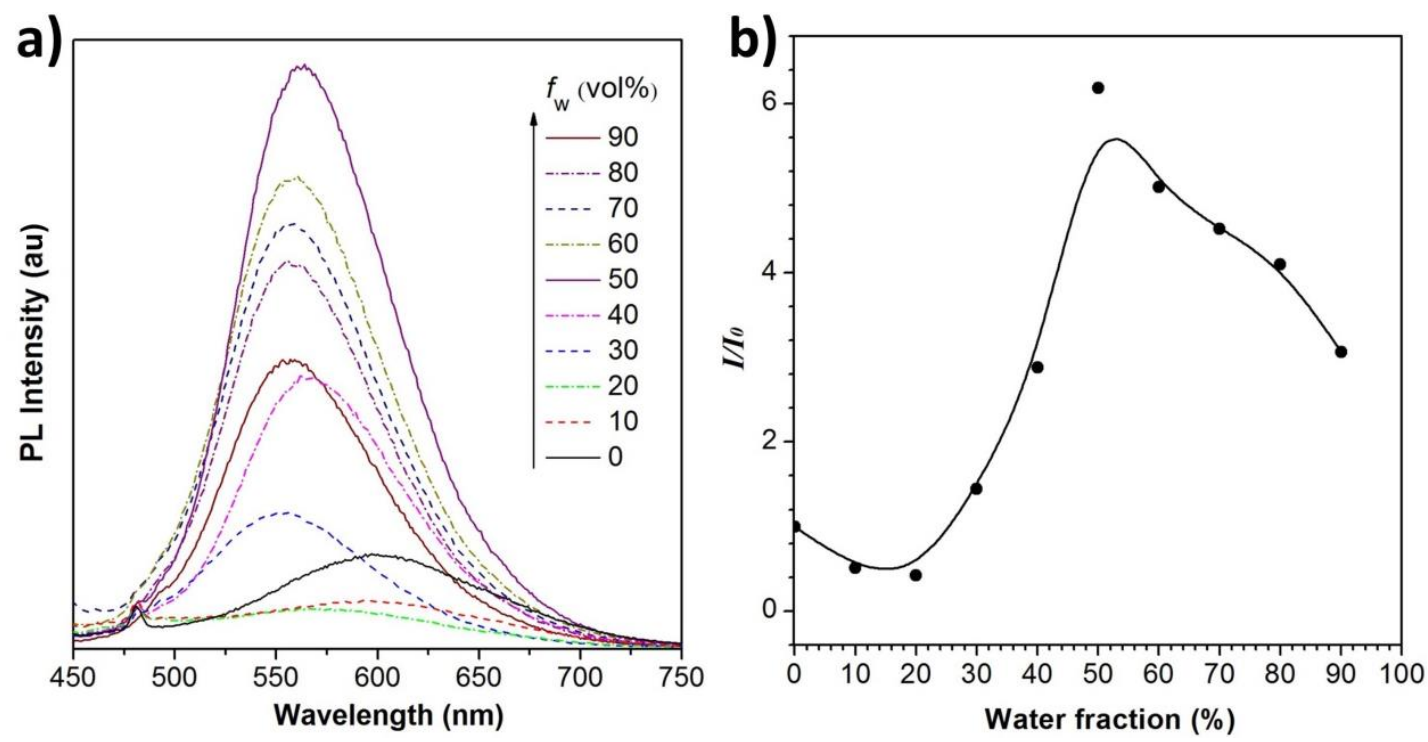

Figure S9. (a) Photoluminescence (PL) spectra of N-DO3AtBu $(10 \mu \mathrm{M})$ in mixtures of $\mathrm{DMSO} /$ water with different water fraction $\left(f_{\mathrm{w}}\right)$. (b) Plots of the relative emission intensity of $\mathrm{N}-\mathrm{DO} 3 \mathrm{~A} t \mathrm{Bu}$ versus water fraction. $I_{0}$ and $I$ are the peak values of PL intensities of $\mathrm{N}$ $\mathrm{DO} 3 \mathrm{~A} t \mathrm{Bu}(10 \mu \mathrm{M})$ in DMSO and DMSO/water mixtures, respectively. 

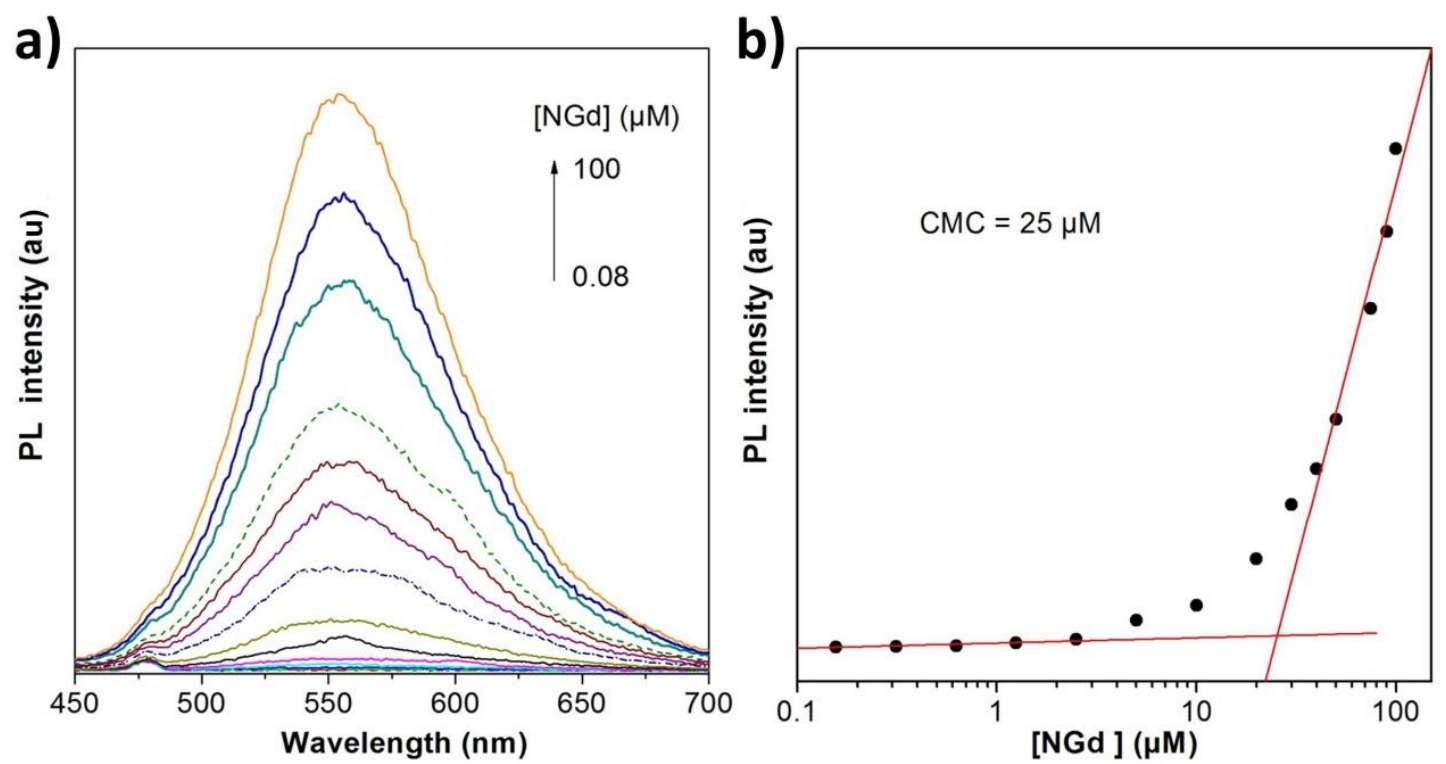

Figure S10. Critical micelle concentration (CMC) of NGd. Plotting the fluorescence intensity (a) versus the dye concentration generates two lines (b), the intersection of which gives the $\mathrm{CMC}$ of $25 \mu \mathrm{M}$.

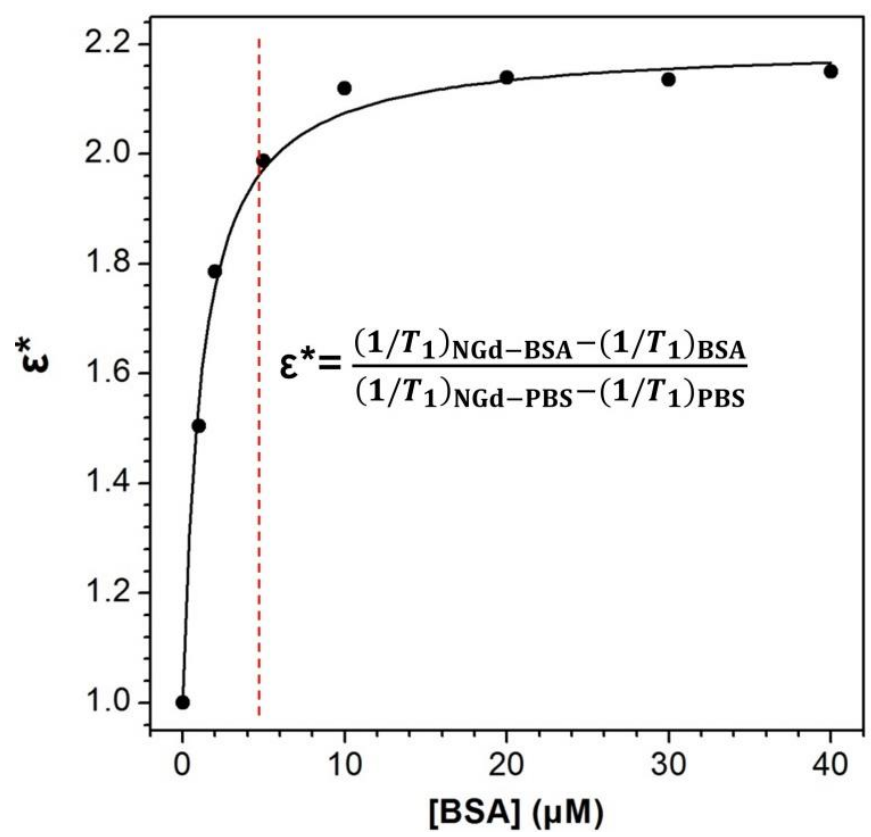

Figure S11. Relaxation enhancement curve of NGd with the increase of BSA concentration. $\varepsilon^{*}$ is the enhanced factor, and the concentration of NGd is $10 \mu \mathrm{M}$. The red dotted line is the mark of the molar ratio of BSA/NGd of 1:2, which is as the initial feed ratio to synthesize the NGd-ACs. 

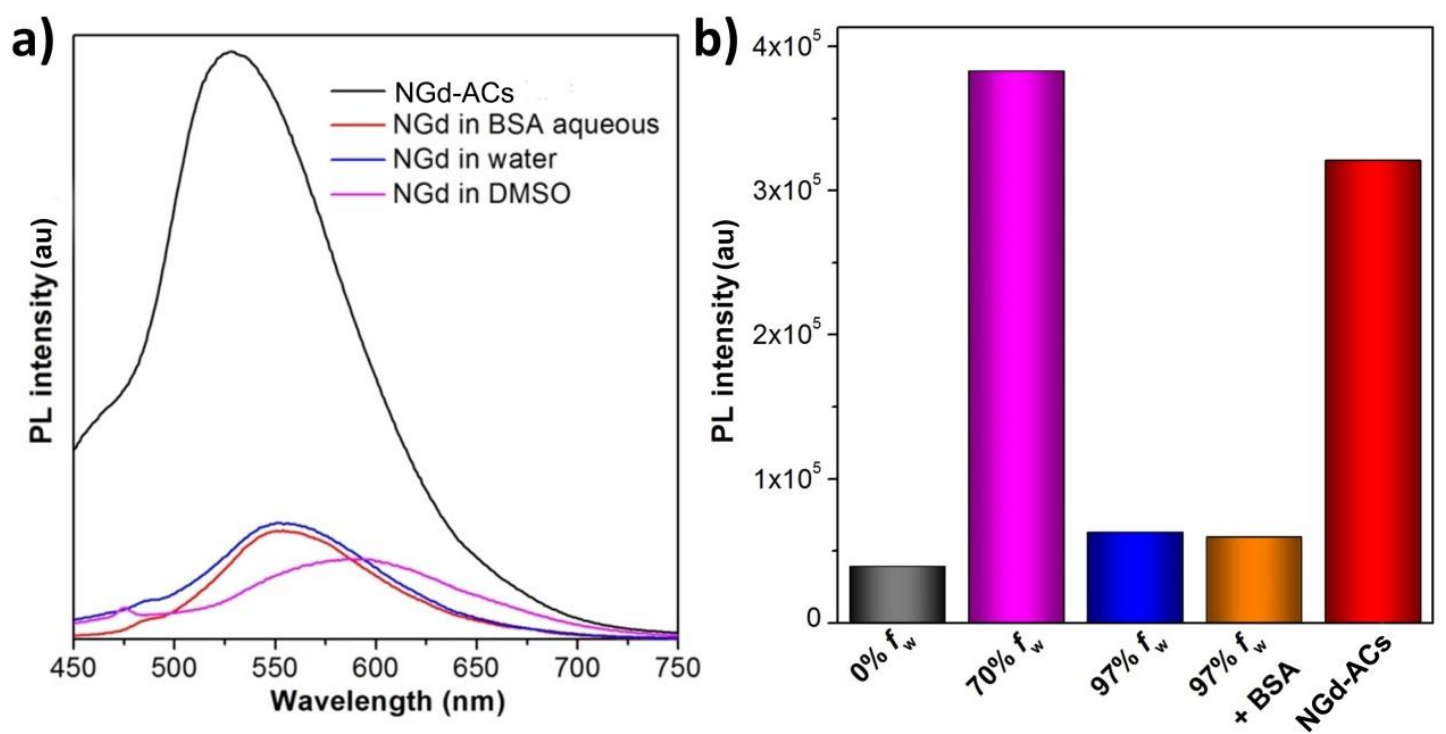

Figure S12. (a) Photoluminescence intensity of NGd-ACs, NGd molecules in DMSO, water, BSA aqueous solution (the molar ratio of $\mathrm{NGd} / \mathrm{BSA}=2: 1$ ), respectively. (b) Comparison of photoluminescence (PL) intensity of NGd $(10 \mu \mathrm{M})$ in mixtures of water/DMSO with $0,70 \%$, $97 \%, 97 \%$ (BSA) water fraction $\left(f_{\mathrm{w}}\right)$, respectively, and NGd-ACs $(10 \mu \mathrm{M})$.

Table S1. Maximum emission, PL intensity and quantum yield $\left(\Phi_{F}\right)$ of NGd-ACs, and NGd in different solvents (BSA aqueous, Deionized water, and DMSO), respectively.

\begin{tabular}{lll}
\hline & $\lambda_{\mathrm{em}}[\mathrm{nm}]$ & $\Phi_{\mathrm{F}}[\%]$ \\
\hline NGd-ACs & 529 & $14.2 \pm 0.1$ \\
NGd in BSA aqueous & 552 & $2.4 \pm 0.2$ \\
NGd in water & 551 & $2.0 \pm 0.2$ \\
NGd in DMSO & 594 & $4.1 \pm 0.1$ \\
\hline
\end{tabular}



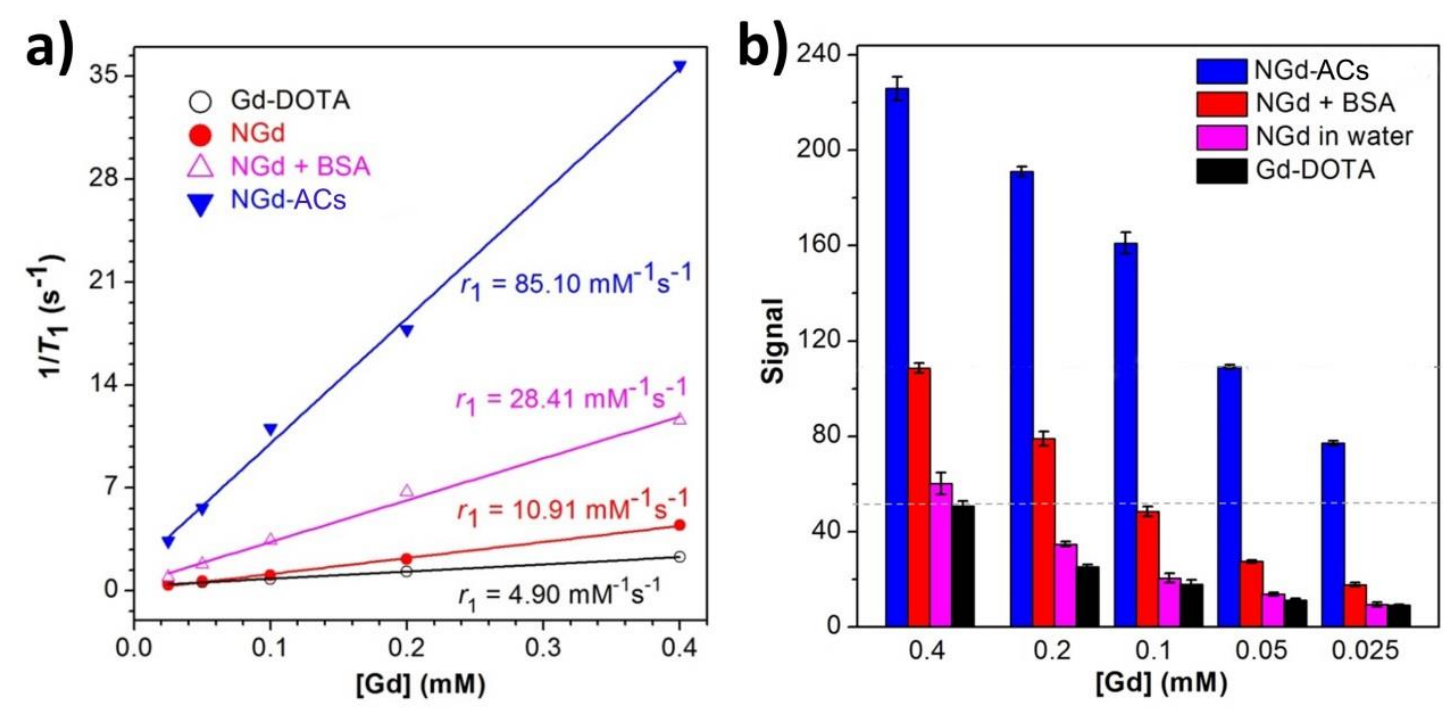

Figure S13. (a) $T_{1}$ relaxivities of NGd-ACs, NGd in BSA aqueous solution (the molar ratio of $\mathrm{NGd} / \mathrm{BSA}=2: 1$ ), NGd in water, and Gd-DOTA at $0.5 \mathrm{~T}$, respectively. (b) The signals of phantom images of NGd-ACs, NGd in BSA aqueous, NGd in water, and Gd-DOTA at different Gd(III) concentrations measured by ImageJ, respectively.

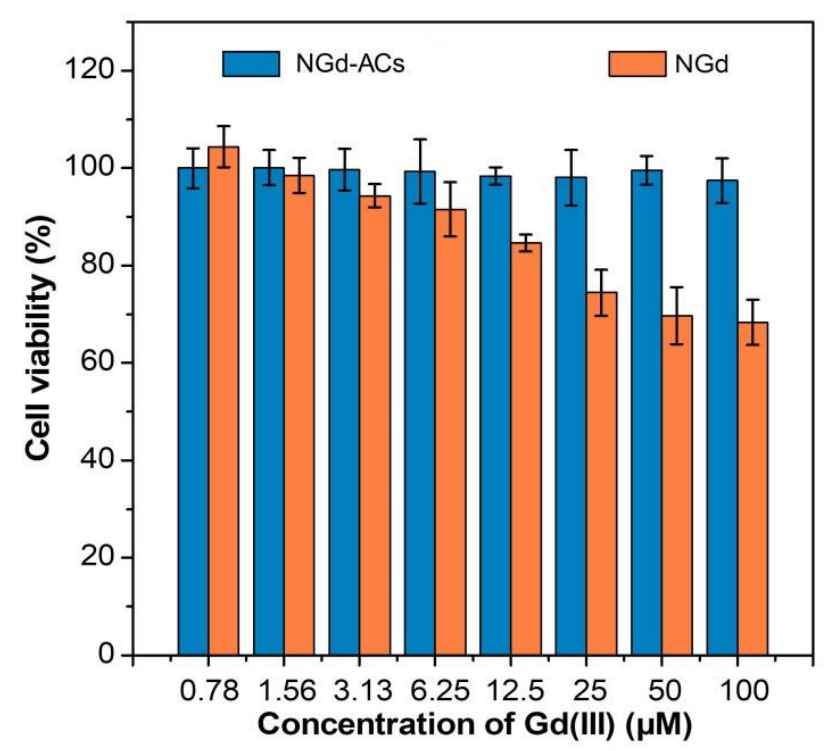

Figure S14. Cell viability of NGd-ACs and NGd by MTT. 
Reference

[1] P. Wei, L. Duan, D. Zhang, J. Qiao, L. Wang, R. Wang, G. Dong, Y. Qiu, J. Mater. Chem. 2008, 18,806 .

[2] Y.-S. Yen, J.-S. Ni, W.-I. Hung, C.-Y. Hsu, H.-H. Chou, J.-T. s. Lin, ACS Appl. Mater. Inter. 2016, $8,6117$.

[3] a) L. Wang, H. Lin, L. Ma, J. Jin, T. Shen, R. Wei, X. Wang, H. Ai, Z. Chen, J. Gao, Nanoscale 2017, 9, 4516; b) L. Wang, H. Lin, L. Ma, C. Sun, J. Huang, A. Li, T. Zhao, Z. Chen, J. Gao, J. Mater. Chem. B 2017, 5, 8004.

[4] A. C. Esqueda, J. A. Lopez, G. Andreu-de-Riquer, J. C. Alvarado-Monzon, J. Ratnakar, A. J. Lubag, A. D. Sherry, L. M. De Leon-Rodriguez, J. Am. Chem. Soc. 2009, 131, 11387.

[5] J. Y. Jun, H. H. Nguyen, S.-Y.-R. Paik, H. S. Chun, B.-C. Kang, S. Ko, Food Chemistry 2011, 127, 1892. 